\title{
Asymptotic normality of Huber-Dutter estimators in a linear EV model with AR(1) processes
}

\author{
Hongchang $\mathrm{Hu}^{1 *}$ and Xiong Pan ${ }^{2}$
}

\section{"Correspondence:}

retutome@163.com

'School of Mathematics and

Statistics, Hubei Normal University,

Huangshi, 435002, China

Full list of author information is

available at the end of the article

\begin{abstract}
The paper studies a linear errors-in-variables model with first order autoregressive processes. The Huber-Dutter (HD) estimators of unknown parameters are given, and the asymptotic normality of the HD estimators is investigated. Finally, a simple example is given to illustrate our estimation method.
\end{abstract}

MSC: 60F05; 60G10; 62F35; 62M10; 60G42

Keywords: linear errors-in-variables model; Huber-Dutter estimator; autoregressive processes; asymptotic normality

\section{Introduction}

Consider the following linear errors-in-variables (EV) model:

$$
\left\{\begin{array}{l}
y_{t}=x_{t}^{T} \beta+\varepsilon_{t}, \\
X_{t}=x_{t}+\zeta_{t}, \quad t=1,2, \ldots, n,
\end{array}\right.
$$

where the superscript $T$ denotes the transpose throughout the paper, $\left\{y_{t}, t=1,2, \ldots, n\right\}$ are scalar response variables, $\left\{X_{t}=\left(X_{t 1}, X_{t 2}, \ldots, X_{t d}\right)^{T}, t=1,2, \ldots, n\right\}$ and $\left\{x_{t}=\left(x_{t 1}, x_{t 2}, \ldots\right.\right.$, $\left.\left.x_{t d}\right)^{T}, t=1,2, \ldots, n\right\}$ are observable and unobservable random variables, respectively, $\beta=$ $\left(\beta_{1}, \ldots, \beta_{d}\right)^{T}$ is a vector of $d$ unknown parameters, $\left\{\zeta_{t}\right\}$ are independent and identically distributed (i.i.d.) measurement errors with $E \zeta_{t}=0$ and $\operatorname{Var}\left(\zeta_{t}\right)=\sigma_{\zeta}^{2} I_{d},\left\{\zeta_{t}\right\}$ and $\left\{\varepsilon_{t}\right\}$ are independent, $\left\{\left(\varepsilon_{t}, \zeta_{t}^{T}\right)^{T}\right\}$ and $\left\{x_{t}\right\}$ are independent, and $\left\{\varepsilon_{t}, t=1,2, \ldots, n\right\}$ are the first order autoregressive $(\mathrm{AR}(1))$ processes

$$
\varepsilon_{1}=\eta_{1}, \quad \varepsilon_{t}=a \varepsilon_{t-1}+\eta_{t}, \quad t=2,3, \ldots, n,
$$

where $\left\{\eta_{t}, t=1,2, \ldots, n\right\}$ are i.i.d. random errors with zero mean and finite variance $\sigma^{2}>0$, and $-\infty<a<\infty$ is a one-dimensional unknown parameter. A common assumption is that the ratio of the error variances $\lambda=\frac{\sigma^{2}}{\sigma_{\zeta}^{2}}$ is known. This is assumed throughout this paper and all variables are assumed scaled so that $\lambda=1$.

The linear errors-in-variables model (1.1) with AR(1) processes (1.2) includes three important special models: (1) an ordinary linear regression model with $\mathrm{AR}(1)$ processes (when $\zeta_{t}=0$, see e.g., Hu [1], Maller [2], Pere [3], and Fuller [4]); (2) an ordinary linear 
errors-in-variables model (when $a=0$, see e.g., Miao and Liu [5], Miao et al. [6, 7], Liu and Chen [8], Cui [9], Cui and Chen [10], Cheng and Van Ness [11]); (3) autoregressive processes (when $\beta=0$, see e.g., Hamilton [12], Brockwell and Davis [13], and Fuller [4]). The independence assumption for the errors is not always valid in applications, especially for sequentially collected economic data, which often exhibit evident dependence in the errors. Recently, linear errors-in-variables models with serially correlated errors have attracted increasing attention from statisticians; see, for example, Baran [14], Fan et al. [15], Miao et al. [16], among others.

It is well known that in the EV model, the ordinary least-squares (OLS) estimators are biased and inconsistent and that orthogonal regression is better in that case Fuller [17]. However, both methods are very sensitive to outliers in the data and some robust alternatives have been proposed. Brown [18] and Ketellapper and Ronner [19] applied robust ordinary regression techniques in the EV model. Zamar [20] proposed robust orthogonal regression $M$-estimators and showed that it outperformed the robust ordinary regression. Cheng and Van Ness [21] generalized the proposal of Zamar by defining robust orthogonal Generalized $M$-estimators which had bounded influence function in the simple case. He and Liang [22] proposed a regression quantile approach in the EV model which allowed for heavier tailed errors distribution than the gaussian distribution. Fekri and Ruiz-Gazen [23] proposed robust weighted orthogonal regression.

Over the last 40 years, several estimators in linear regression models that posses robustness have been proposed, such as Wu [24], Silvapullé [25], Hampel et al. [26], Huber and Ronchetti [27], Li [28], Babu [29], Cheng and Van Ness [21], Salibian-Barrera and Zamar [30], Wu and Wang [31], Zhou and Wu [32], and so on. It is well known that HD estimate approach is one of important robust techniques. Recently, some authors applied HD estimate approach to regression models. For example, Silvapullé [25] established asymptotic normality of HD estimators for the linear regression model with i.i.d. errors. $\mathrm{Hu}$ [1] investigated asymptotic normality of HD estimators for the linear regression model with AR(1) errors. Tong et al. [33] considered consistency and normality of HD estimators for the partial linear regression model. However, nobody used the HD method to investigate the models (1.1)-(1.2).

The paper discusses the models (1.1)-(1.2) with a robust approach, which has been suggested by Huber and Dutter. We extend some results of $\mathrm{Hu}$ [1], Silvapullé [25], etc. to the EV regression model with $\mathrm{AR}(1)$ errors. The organization of the paper is as follows. In Section 2 estimators of $\beta, a$ and $\sigma^{2}$ are given by HD method. Under general conditions, the asymptotic normality of the HD estimators is investigated in Section 3. The theoretical proofs of main results are presented in Section 4, a simple example is given in Section 5 .

\section{Estimation method}

By (1.2), we have

$$
\varepsilon_{t}=\sum_{j=1}^{t} a^{t-j} \eta_{j}, \quad t=1,2, \ldots
$$


thus $\varepsilon_{t}$ is measurable with respect to the $\sigma$-field $H$ generated by $\eta_{1}, \eta_{2}, \ldots, \eta_{t}, E \varepsilon_{t}=0$ and

$$
\operatorname{Var}\left(\varepsilon_{t}\right)= \begin{cases}\sigma^{2}\left(\frac{1-a^{2 t}}{1-a^{2}}\right), & \text { if }|a| \neq 1 \\ \sigma^{2} t, & \text { if }|a|=1\end{cases}
$$

Furthermore,

$$
\begin{aligned}
\Delta_{n}(a, \sigma) & =\sum_{t=2}^{n} E \varepsilon_{t-1}^{2}= \begin{cases}\sigma^{2}\left(\frac{a^{2 n}-a^{2}+(n-1)\left(1-a^{2}\right)}{\left(1-a^{2}\right)^{2}}\right), & \text { if }|a| \neq 1, \\
\frac{1}{2} \sigma^{2} n(n-1), & \text { if }|a|=1,\end{cases} \\
& = \begin{cases}O(n), & \text { if }|a|<1, \\
O\left(a^{2 n}\right), & \text { if }|a|>1, \\
O\left(n^{2}\right), & \text { if }|a|=1 .\end{cases}
\end{aligned}
$$

Let $y_{0}=0, x_{0}=0$. From (1.1),

$$
\varepsilon_{t}=y_{t}-\left(X_{t}^{T}-\zeta_{t}^{T}\right) \beta
$$

By the above equation and (1.2), we obtain

$$
\begin{aligned}
\eta_{t} & =\varepsilon_{t}-a \varepsilon_{t-1} \\
& =y_{t}-X_{t}^{T} \beta+\zeta_{t}^{T} \beta-a\left(y_{t-1}-X_{t-1}^{T} \beta+\zeta_{t-1}^{T} \beta\right) .
\end{aligned}
$$

Thus we could consider HD estimators by minimizing

$$
Q\left(\beta^{T}, \sigma, a\right)=\sum_{t=1}^{n} \rho\left(\frac{y_{t}-X_{t}^{T} \beta+\zeta_{t}^{T} \beta-a\left(y_{t-1}-X_{t-1}^{T} \beta+\zeta_{t-1}^{T} \beta\right)}{\sigma}\right) \sigma+A_{n} \sigma
$$

where $\rho \geq 0$ is convex, $\rho(0)=0, \frac{\rho(t)}{|t|} \rightarrow k$ as $|t| \rightarrow \infty$ for some $k>0$, and $\left\{A_{n}\right\}$ is a suitably chosen sequence of constants.

Remark 1 Since

$$
\operatorname{Var}\left(\frac{y_{t}-X_{t}^{T} \beta-a\left(y_{t-1}-X_{t-1}^{T} \beta\right)}{\sqrt{1+\left(1+a^{2}\right) \beta^{T} \beta}}\right)=\sigma^{2},
$$

using the method of HD, we also obtain HD estimators by minimizing

$$
\tilde{Q}\left(\beta^{T}, \sigma, a\right)=\sum_{t=1}^{n} \rho\left(\frac{\frac{y_{t}-X_{t}^{T} \beta-a\left(y_{t-1}-X_{t-1}^{T} \beta\right)}{\sqrt{1+\left(1+a^{2}\right) \beta^{T} \beta}}}{\sigma}\right) \sigma+A_{n} \sigma .
$$

We will investigate its estimators in the future because there are some difficulties. For example, there is very sophisticated calculation, and it is difficult to investigate the asymptotic properties of these unknown parametric estimators because

$$
y_{t}-X_{t}^{T} \beta-a\left(y_{t-1}-X_{t-1}^{T} \beta\right)=\eta_{t}-\left(\zeta_{t}^{T}-a \zeta_{t-1}^{T}\right) \beta
$$

are dependent. 
Let us introduce some notation: $1 \times(d+2)$ vector $\theta=\left(\beta^{T}, \sigma, a\right)$ and its estimator $\hat{\theta}_{n}=$ $\left(\hat{\beta}_{n}^{T}, \hat{\sigma}_{n}, \hat{a}_{n}\right)$. For an arbitrary function $f, f^{\prime}$ and $f^{\prime \prime}$ are the first and second derivatives of $f$, respectively. $\|x\|$ is the Euclidean norm of $x$ and $\varepsilon_{0}=0 .(M)_{i j}$ and $(U)_{i}$ are the $(i, j)$ th component of matrix $M$ and the $i$ th component of vector $U$, respectively.

The HD estimators for $\theta$ are obtained by solving the estimating equations by equating to 0 the derivatives

$$
\begin{aligned}
\frac{\partial Q}{\partial \beta^{T}} & =-\sum_{t=1}^{n} \psi\left(\frac{y_{t}-X_{t}^{T} \beta+\zeta_{t}^{T} \beta-a\left(y_{t-1}-X_{t-1}^{T} \beta+\zeta_{t-1}^{T} \beta\right)}{\sigma}\right)\left(X_{t}-a X_{t-1}-\left(\zeta_{t}-a \zeta_{t-1}\right)\right) \\
& =-\sum_{t=1}^{n} \psi\left(\frac{\varepsilon_{t}-a \varepsilon_{t-1}}{\sigma}\right)\left(X_{t}-a X_{t-1}-\left(\zeta_{t}-a \zeta_{t-1}\right)\right) \\
\frac{\partial Q}{\partial \sigma} & =-\sum_{t=1}^{n}\left\{\psi\left(\frac{\varepsilon_{t}-a \varepsilon_{t-1}}{\sigma}\right) \frac{\varepsilon_{t}-a \varepsilon_{t-1}}{\sigma}-\rho\left(\frac{\varepsilon_{t}-a \varepsilon_{t-1}}{\sigma}\right)\right\}+A_{n} \\
& =-\left\{\sum_{t=1}^{n} \chi\left(\frac{\varepsilon_{t}-a \varepsilon_{t-1}}{\sigma}\right)-A_{n}\right\}
\end{aligned}
$$

and

$$
\frac{\partial Q}{\partial a}=-\sum_{t=1}^{n} \psi\left(\frac{\varepsilon_{t}-a \varepsilon_{t-1}}{\sigma}\right) \varepsilon_{t-1},
$$

where $\psi=\rho^{\prime}$ and $\chi(u)=u \psi(u)-\rho(u)=\int_{0}^{u} x d \psi(x)$.

The corresponding estimators, if they exist (see Proposition 2.1), will satisfy

$$
\begin{aligned}
& \sum_{t=1}^{n} \psi\left(\frac{\hat{\varepsilon}_{t}-\hat{a}_{n} \hat{\varepsilon}_{t-1}}{\hat{\sigma}_{n}}\right)\left(X_{t}-\hat{a}_{n} X_{t-1}-\left(\zeta_{t}-\hat{a}_{n} \zeta_{t-1}\right)\right)=0, \\
& \sum_{t=1}^{n} \chi\left(\frac{\hat{\varepsilon}_{t}-\hat{a}_{n} \hat{\varepsilon}_{t-1}}{\hat{\sigma}_{n}}\right)=A_{n}
\end{aligned}
$$

and

$$
\sum_{t=1}^{n} \psi\left(\frac{\hat{\varepsilon}_{t}-\hat{a}_{n} \hat{\varepsilon}_{t-1}}{\hat{\sigma}_{n}}\right) \hat{\varepsilon}_{t-1}=0
$$

with $\hat{\varepsilon}_{t}=y_{t}-x_{t}^{T} \hat{\beta}_{n}$.

Although $\left\{\zeta_{t}\right\}$ are unknown in (2.9)-(2.11), but we easily estimate it by the method of Fuller [17] in practice.

In what follows, it will be assumed that $n \geq d+2$ and $A_{n}>0$. Without loss of generality, we may assume $k=1$. (Its definition has been given between (2.5) and Remark1.) Therefore $\psi$ is bounded and increases from -1 to +1 . It will also be assumed that $\chi$ is bounded.

Remark 2 From the above equations, it is easily seen that our estimators include some existing estimators; see, for example, simultaneous $M$-estimators of the location and the scale $\left(a=0, A_{n}=0\right)$, the least-squares estimators $\left(\rho(u)=u^{2}, A_{n}=0\right.$, and $\left.\sigma=1\right)$, the least absolute deviation estimators $\left(\rho(u)=|u|, A_{n}=0\right)$. In particular, we discuss three important cases as follows. 
Case 1. Let $a=0, \zeta_{t}=0$. The estimating equations (2.9)-(2.11) may be written as

$$
\sum_{t=1}^{n} \psi\left(\frac{y_{t}-X_{t}^{T} \hat{\beta}_{n}}{\hat{\sigma}_{n}}\right) X_{t}=0, \quad \sum_{t=1}^{n} \chi\left(\frac{y_{t}-X_{t}^{T} \hat{\beta}_{n}}{\hat{\sigma}_{n}}\right)=A_{n}
$$

which are the same as Silvapullés [25].

Case 2. If $\rho(u)=|u|^{q}(0<q \neq 1), A_{n}=0$, and $\zeta_{t}=0$, then the above equations (2.9)-(2.11) may be rewritten as

$$
\begin{aligned}
& \sum_{t=1}^{n}\left|\frac{\hat{\varepsilon}_{t}-\hat{a}_{n} \hat{\varepsilon}_{t-1}}{\hat{\sigma}_{n}}\right|^{q-2} \frac{\hat{\varepsilon}_{t}-\hat{a}_{n} \hat{\varepsilon}_{t-1}}{\hat{\sigma}_{n}}\left(X_{t}-\hat{a}_{n} X_{t-1}\right)=0, \\
& \sum_{t=1}^{n}\left|\frac{\hat{\varepsilon}_{t}-\hat{a}_{n} \hat{\varepsilon}_{t-1}}{\hat{\sigma}_{n}}\right|^{q}=0,
\end{aligned}
$$

and

$$
\sum_{t=1}^{n}\left|\frac{\hat{\varepsilon}_{t}-\hat{a}_{n} \hat{\varepsilon}_{t-1}}{\hat{\sigma}_{n}}\right|^{q-2} \frac{\hat{\varepsilon}_{t}-\hat{a}_{n} \hat{\varepsilon}_{t-1}}{\hat{\sigma}_{n}} \hat{\varepsilon}_{t-1}=0
$$

with $\hat{\varepsilon}_{t}=y_{t}-X_{t}^{T} \hat{\beta}_{n}$.

Let $a=0$ and $\rho(u)=|u|^{q}(0<q \neq 1), A_{n}=0$. We rewrite (2.13)-(2.15) by

$$
\sum_{t=1}^{n}\left|\frac{y_{t}-X_{t}^{T} \hat{\beta}_{n}}{\hat{\sigma}_{n}}\right|^{q-2} \frac{y_{t}-X_{t}^{T} \hat{\beta}_{n}}{\hat{\sigma}_{n}}=0, \quad \sum_{t=1}^{n}\left|\frac{y_{t}-X_{t}^{T} \hat{\beta}_{n}}{\hat{\sigma}_{n}}\right|^{q}=0 .
$$

Furthermore, if $\sigma$ is a constant, then the estimator of $\beta$ satisfies the following equation:

$$
\sum_{t=1}^{n}\left|y_{t}-X_{t}^{T} \hat{\beta}_{n}\right|^{q-2}\left(y_{t}-X_{t}^{T} \hat{\beta}_{n}\right)=0
$$

which is $L_{q}$ or the maximum likelihood estimate equation for the parameter $\beta$ in a linear regression model with $q$-norm distribution errors. Many authors have investigated (2.17), such as Arcones [34] and Zeckhauser and Thompson [35], Ronner [36, 37], and so on.

Case 3. Let $\zeta_{t}=0$. The estimating equations (2.9)-(2.11) may be written as

$$
\begin{aligned}
& \sum_{t=1}^{n} \psi\left(\frac{\hat{\varepsilon}_{t}-\hat{a}_{n} \hat{\varepsilon}_{t-1}}{\hat{\sigma}_{n}}\right)\left(X_{t}-\hat{a}_{n} X_{t-1}\right)=0, \\
& \sum_{t=1}^{n} \chi\left(\frac{\hat{\varepsilon}_{t}-\hat{a}_{n} \hat{\varepsilon}_{t-1}}{\hat{\sigma}_{n}}\right)=A_{n},
\end{aligned}
$$

and

$$
\sum_{t=1}^{n} \psi\left(\frac{\hat{\varepsilon}_{t}-\hat{a}_{n} \hat{\varepsilon}_{t-1}}{\hat{\sigma}_{n}}\right) \hat{\varepsilon}_{t-1}=0
$$

with $\hat{\varepsilon}_{t}=y_{t}-x_{t}^{T} \hat{\beta}_{n}$, which are the same as Hu's [1]. 
From Proposition 1 in Silvapullé [25] and pp.136 in Huber and Ronchetti [27], the existence results of the HD estimators may be given by the following.

Proposition 2.1 Suppose that $\rho^{\prime \prime}$ is continuous and, for some $A>0, v<1-A V^{-1}$, where $v$ is the largest jump in the error distribution, $V=\chi(-\infty) \wedge \chi(\infty)$, then the equation $E\left\{\psi\left(\frac{\varepsilon-\mu}{\sigma}\right), \chi\left(\frac{\varepsilon-\mu}{\sigma}\right)-A\right\}=0$ has a solution $(\mu(A), \sigma(A))$ with $\sigma(A)>0$. Especially, when $A=\lim _{n \rightarrow \infty} n^{-1} A_{n}$, where $A_{n}$ is defined in (2.5), we denote it by $(\mu, \sigma)$ with $\sigma>0$.

\section{Main results}

To obtain our results, we start with some assumptions.

(A1) $\max _{t=1}^{n}\left\|X_{t}\right\|<\infty$.

(A2) $\lim _{n \rightarrow \infty} n^{\frac{1}{2}}\left(n^{-1} A_{n}-A\right)=0$ for some $0<n^{-1} A_{n}, A<\min \{\chi(\infty), \chi(-\infty)\}$.

(A3) The function $\psi^{\prime \prime}$ is continuous.

(A4) $E \psi\left(\frac{\eta_{t}}{\sigma}\right)=0$ for any $\sigma>0 . b=E \psi^{\prime}\left(\frac{\eta_{t}}{\sigma}\right)>0, c=E\left\{\psi^{\prime}\left(\frac{\eta_{t}}{\sigma}\right) \frac{\eta_{t}}{\sigma}\right\}, r=E\left\{\psi^{\prime}\left(\frac{\eta_{t}}{\sigma}\right) \eta_{t}^{2}\right\}, b r \geq c^{2}$, $\operatorname{Var}\left(\psi^{\prime}\left(\frac{\eta_{t}}{\sigma}\right)\right)<\infty, \operatorname{Var}\left(\psi^{\prime}\left(\frac{\eta_{t}}{\sigma}\right) \eta_{t}\right)<\infty, \operatorname{Var}\left(\psi^{\prime}\left(\frac{\eta_{t}}{\sigma}\right) \eta_{t}^{2}\right)<\infty, E \eta_{t}^{6}<\infty$ and $E\left(\psi^{\prime \prime}\left(\frac{\eta_{t}}{\sigma}\right)\right)^{2}<\infty$.

(A5) For any $a \in(-\infty, \infty), X_{n}(a)=\sum_{t=1}^{n}\left(X_{t}-a X_{t-1}\right)\left(X_{t}-a X_{t-1}\right)^{T}$ is positive definite for sufficiently large $n$.

Remark 3 The condition (A1) is often imposed in the estimation theory of regression models. The condition (A2) is used by Tong et al. [33]. In addition, by (A1) and (A2), we can obtain conditions $n^{-1} \max _{t=1}^{n}\left\|X_{t}\right\|^{2} \rightarrow 0$ and $\lim _{n \rightarrow \infty}\left(n^{-1} A_{n}-A\right)=0$, which are used by Silvapullé [25]. The conditions (A3) and (A4) except $E \eta_{t}^{6}<\infty$ and $E\left(\psi^{\prime \prime}\left(\frac{\eta_{t}}{\sigma}\right)\right)^{2}<\infty$ are used by Silvapullé [25]. The condition (A5) is used by Maller [2], Hu [1], etc. Therefore, our conditions are quite mild and can easily be satisfied.

For ease of exposition, we shall introduce the following notations which will be used later in the paper. Define

$$
\begin{aligned}
& \Theta_{1}=\left\{\tilde{\theta}:|\tilde{\theta}-\theta| \leq C n^{-\frac{1}{2}}\right\}, \\
& \Theta_{2}=\left\{\tilde{\theta}:|\tilde{\beta}-\beta| \leq C n^{-\frac{1}{2}},|\tilde{\sigma}-\sigma| \leq C n^{-\frac{1}{2}},|\tilde{a}-a| \leq C n^{-1}\right\}, \\
& \Theta_{3}=\left\{\tilde{\theta}:|\tilde{\beta}-\beta| \leq C n^{-\frac{1}{2}},|\tilde{\sigma}-\sigma| \leq C n^{-\frac{1}{2}},|\tilde{a}-a| \leq C a^{-\frac{3 n}{2}}\right\}, \\
& S_{n}(\theta)=\frac{\partial Q}{\partial \theta}=\left(\frac{\partial Q}{\partial \beta^{T}}, \frac{\partial Q}{\partial \sigma}, \frac{\partial Q}{\partial a}\right)=\left(\left[S_{n}(\theta)\right]_{\beta, \sigma}, \frac{\partial Q}{\partial a}\right),
\end{aligned}
$$

and

$$
F_{n}(\theta)=\frac{\partial^{2} Q}{\partial \theta \partial \theta^{T}}=\left(\begin{array}{ccc}
\frac{\partial^{2} Q}{\partial \beta^{T} \partial \beta} & \frac{\partial^{2} Q}{\partial \beta^{T} \partial \sigma} & \frac{\partial^{2} Q}{\partial \beta^{T} \partial a} \\
* & \frac{\partial^{2} Q}{\partial \sigma^{2}} & \frac{\partial^{2} Q}{\partial \sigma \partial a} \\
* & * & \frac{\partial^{2} Q}{\partial a^{2}}
\end{array}\right),
$$


where the $*$ indicates that the elements are filled in by symmetry, and

$$
\begin{aligned}
& \frac{\partial^{2} Q}{\partial \beta^{T} \partial \beta}= \frac{1}{\sigma} \sum_{t=1}^{n} \psi^{\prime}\left(\frac{\varepsilon_{t}-a \varepsilon_{t-1}}{\sigma}\right) \\
& \cdot\left(X_{t}-a X_{t-1}-\left(\zeta_{t}-a \zeta_{t-1}\right)\right)\left(X_{t}-a X_{t-1}-\left(\zeta_{t}-a \zeta_{t-1}\right)\right)^{T} \\
&= X_{n}(a, \omega), \\
& \frac{\partial^{2} Q}{\partial \beta^{T} \partial \sigma}= \frac{1}{\sigma^{2}} \sum_{t=1}^{n} \psi^{\prime}\left(\frac{\varepsilon_{t}-a \varepsilon_{t-1}}{\sigma}\right)\left(\varepsilon_{t}-a \varepsilon_{t-1}\right)\left(X_{t}-a X_{t-1}-\left(\zeta_{t}-a \zeta_{t-1}\right)\right)^{T}, \\
& \frac{\partial^{2} Q}{\partial \beta^{T} \partial a}= \sum_{t=1}^{n} \psi^{\prime}\left(\frac{\varepsilon_{t}-a \varepsilon_{t-1}}{\sigma}\right) \frac{\varepsilon_{t-1}}{\sigma}\left(X_{t}-a X_{t-1}-\left(\zeta_{t}-a \zeta_{t-1}\right)\right)^{T} \\
&+\sum_{t=1}^{n} \psi\left(\frac{\varepsilon_{t}-a \varepsilon_{t-1}}{\sigma}\right)\left(X_{t-1}-\zeta_{t-1}\right)^{T}, \\
& \frac{\partial^{2} Q}{\partial \sigma^{2}}=\frac{1}{\sigma^{2}} \sum_{t=1}^{n} \psi^{\prime}\left(\frac{\varepsilon_{t}-a \varepsilon_{t-1}}{\sigma}\right)\left(\varepsilon_{t}-a \varepsilon_{t-1}\right)^{2}, \\
& \frac{\partial^{2} Q}{\partial \sigma \partial a}=\frac{1}{\sigma^{2}} \sum_{t=1}^{n} \psi^{\prime}\left(\frac{\varepsilon_{t}-a \varepsilon_{t-1}}{\sigma}\right)\left(\varepsilon_{t}-a \varepsilon_{t-1}\right) \varepsilon_{t-1},
\end{aligned}
$$

and

$$
\frac{\partial^{2} Q}{\partial a^{2}}=\frac{1}{\sigma} \sum_{t=1}^{n} \psi^{\prime}\left(\frac{\varepsilon_{t}-a \varepsilon_{t-1}}{\sigma}\right) \varepsilon_{t-1}^{2}
$$

Theorem 3.1 Suppose that conditions (A1)-(A5) hold. Then, as $n \rightarrow \infty$ :

(1) For $|a|<1$ and $\theta \in \Theta_{1}$, we have

$$
\left(\hat{\theta}_{n}-\theta\right) D_{n}(\theta) \operatorname{Var}^{-\frac{1}{2}}\left(S_{n}(\theta)\right) \rightarrow_{D} N\left(0, I_{d+2}\right),
$$

where $D_{n}(\theta)=E\left(F_{n}(\theta)\right)$ and $\operatorname{Var}\left(S_{n}(\theta)\right)$ defined in Lemma 4.1.

(2) For $|a|=1$ and $\theta \in \Theta_{2}$, (3.9) holds.

(3) For $|a|>1$ and $\theta \in \Theta_{3}$, (3.9) holds.

From the above theorem, we may obtain the following corollaries. Here we omit their proofs.

Corollary 3.1 If $\beta=0$ and conditions (A2)-(A5) hold, then

$$
n^{\frac{1}{2}}\left(\hat{\sigma}_{n}-\sigma\right) \rightarrow_{D} N\left(0, \frac{\sigma^{2}}{r^{2}} \operatorname{Var}\left(\chi\left(\frac{\eta_{1}}{\sigma}\right)\right)\right)
$$

and

$$
\Delta_{n}^{\frac{1}{2}}(a, \sigma)\left(\hat{a}_{n}-a\right) \rightarrow_{D} N\left(0, \frac{\sigma^{2}}{b^{2}} E \psi^{2}\left(\frac{\eta_{1}}{\sigma}\right)\right), \quad n \rightarrow \infty
$$


Corollary 3.2 If $a=0$ and conditions (A1)-(A5) hold, then

$$
-\left[S_{n}(\theta)\right]_{\beta, \sigma}\left[\operatorname{Var}^{-\frac{1}{2}} S_{n}(\theta)\right]_{\beta, \sigma} \rightarrow_{D} N\left(0, I_{d+1}\right), \quad n \rightarrow \infty .
$$

Remark 4 Corollary 3.2 is similar to Theorem 2 of Silvapullé [25].

Corollary 3.3 Let $\sigma$ be a constant. If conditions (A1)-(A5) hold, then

$$
\left(\hat{\beta}_{n}-\beta\right)\left(X_{n}(a)+n\left(1+a^{2}\right) \sigma_{\zeta}^{2} I_{d}\right)^{\frac{1}{2}} \rightarrow_{D} N\left(0, \frac{\sigma^{2}}{b^{2}} E \psi^{2}\left(\frac{\eta_{1}}{\sigma}\right) I_{d}\right)
$$

and

$$
\Delta_{n}^{\frac{1}{2}}(a, \sigma)\left(\hat{a}_{n}-a\right) \rightarrow_{D} N\left(0, \frac{\sigma^{2}}{b^{2}} E \psi^{2}\left(\frac{\eta_{1}}{\sigma}\right)\right), \quad n \rightarrow \infty .
$$

Corollary 3.4 Let $\zeta_{t}=0$. If conditions (A1)-(A5) hold, then Theorem 3.1 holds.

Remark 5 For $|a|<1$, Corollary 3.4 is the same as Theorem 3.1 of $\mathrm{Hu}[1]$. Therefore, we extend the corresponding results of $\mathrm{Hu}$ [1] to linear EV models.

If we do not consider the dependency on the parameters $\hat{\beta}_{n}$ and $\hat{\sigma}_{n}$, then we will obtain Theorem 3.2.

Theorem 3.2 Let

$$
[\theta]_{\beta, \sigma}=\left(\beta^{T}, \sigma\right), \quad D_{n}(\theta)=\operatorname{diag}\left(\left[D_{n}(\theta)\right]_{\beta, \sigma}, \frac{b}{\sigma} \Delta_{n}(a, \sigma)\right) .
$$

Suppose that conditions (A1)-(A5) hold. Then

(1) for any $a \in(-\infty, \infty)$,

$$
n^{-\frac{1}{2}}\left[\hat{\theta}_{n}-\theta\right]_{\beta, \sigma}\left[D_{n}(\theta)\right]_{\beta, \sigma} \rightarrow_{D} N(0, \Sigma), \quad n \rightarrow \infty
$$

where

$$
\Sigma=\operatorname{diag}\left\{\Delta_{n}^{-1}(a, \sigma)\left(X_{n}(a)+n\left(1+a^{2}\right) \sigma_{\zeta}^{2} I_{d}\right) E\left(\psi^{2}\left(\frac{\eta_{1}}{\sigma}\right)\right), \operatorname{Var}\left(\chi\left(\frac{\eta_{1}}{\sigma}\right)\right)\right\}
$$

(2) for any $a \in(-\infty, \infty)$,

$$
\Delta_{n}^{\frac{1}{2}}(a, \sigma)\left(\hat{a}_{n}-a\right) \rightarrow_{D} N\left(0, \frac{\sigma^{2}}{b^{2}} E \psi^{2}\left(\frac{\eta_{1}}{\sigma}\right)\right) .
$$

\section{Proofs of main results}

Throughout this paper, let $C$ denote a generic positive constant which could take different value at each occurrence. To prove Theorem 3.1 and Theorem 3.2, we first introduce the following lemmas. 
Lemma 4.1 If (A4) and (A5) hold, then the matrix $D_{n}(\theta)$ is positive definite with $E\left(S_{n}(\theta)\right)=$ 0 for sufficiently large $n$ and

$$
\begin{aligned}
& \operatorname{Var}\left(S_{n}(\theta)\right) \\
& =\left(\begin{array}{ccc}
E \psi^{2}\left(\frac{\eta_{1}}{\sigma}\right)\left(X_{n}(a)+n\left(1+a^{2}\right) \sigma_{\zeta}^{2} I_{d}\right) & \operatorname{Cov}\left(\frac{\partial Q}{\partial \beta^{T}}, \frac{\partial Q}{\partial \sigma}\right) & 0 \\
* & n \operatorname{Var}\left(\chi\left(\frac{\eta_{1}}{\sigma}\right)\right) & 0 \\
0 & 0 & E \psi^{2}\left(\frac{\eta_{1}}{\sigma}\right) \Delta_{n}(a, \sigma)
\end{array}\right) \\
& =\operatorname{diag}\left\{\left[\operatorname{Var}\left(S_{n}(\theta)\right)\right]_{\beta, \sigma}, E \psi^{2}\left(\frac{\eta_{1}}{\sigma}\right) \Delta_{n}(a, \sigma)\right\},
\end{aligned}
$$

where

$$
\operatorname{Cov}\left(\frac{\partial Q}{\partial \beta^{T}}, \frac{\partial Q}{\partial \sigma}\right)=E\left(\psi\left(\frac{\eta_{1}}{\sigma}\right) \chi\left(\frac{\eta_{1}}{\sigma}\right)\right) \sum_{t=1}^{n}\left(X_{t}-a X_{t-1}\right)^{T} .
$$

Furthermore, $\operatorname{Var}\left(S_{n}(\theta)\right)$ is a positive definite matrix.

Proof Note that $E \psi\left(\frac{\eta_{t}}{\sigma}\right)=0, E\left(\varepsilon_{t}\right)=0$, and $\varepsilon_{t}, \zeta_{t}$, and $\eta_{t+1}$ are independent. By (3.3)-(3.8), we easily obtain

$$
D_{n}(\theta)=\left(\begin{array}{ccc}
\frac{b}{\sigma} X_{n}(a)+\frac{n b}{\sigma}\left(1+a^{2}\right) \sigma_{\zeta}^{2} I_{d} & \frac{c}{\sigma} \sum_{t=1}^{n}\left(X_{t}-a X_{t-1}\right) & 0 \\
* & \frac{n r}{\sigma} & 0 \\
0 & 0 & \frac{b}{\sigma} \Delta_{n}(a, \sigma)
\end{array}\right) .
$$

It is easy to show that

$$
\begin{aligned}
& D_{n 1}(\theta)=\left|\frac{b}{\sigma} X_{n}(a)+\frac{n b}{\sigma}\left(1+a^{2}\right) \sigma_{\zeta}^{2} I_{d}\right|>0, \\
& D_{n 2}(\theta)=\left|\begin{array}{cc}
\frac{b}{\sigma} X_{n}(a)+\frac{n b}{\sigma}\left(1+a^{2}\right) \sigma_{\zeta}^{2} I_{d} & \frac{c}{\sigma} \sum_{t=1}^{n}\left(X_{t}-a X_{t-1}\right) \\
* & \frac{n r}{\sigma}
\end{array}\right| \\
& =\left|\frac{b}{\sigma} X_{n}(a)+\frac{n b}{\sigma}\left(1+a^{2}\right) \sigma_{\zeta}^{2} I_{d}\right| \cdot \mid \frac{n r}{\sigma}-\frac{c^{2}}{b \sigma} \sum_{t=1}^{n}\left(X_{t}-a X_{t-1}\right)^{T} \\
& \cdot\left(X_{n}(a)+n\left(1+a^{2}\right) \sigma_{\zeta}^{2} I_{d}\right)^{-1} \sum_{t=1}^{n}\left(X_{t}-a X_{t-1}\right) \mid>0,
\end{aligned}
$$

and

$$
\left|D_{n}(\theta)\right|>0
$$

Thus the matrix $D_{n}(\theta)$ is positive definite.

By (2.6), we have

$$
E\left(\frac{\partial Q}{\partial \beta^{T}}\right)=-\sum_{t=1}^{n} E \psi\left(\frac{\eta_{t}}{\sigma}\right)\left(X_{t}-a X_{t-1}\right)^{T}=0 .
$$


By (2.7) and Proposition 2.1, we have

$$
E\left(\frac{\partial Q}{\partial \sigma}\right)=-\sum_{t=1}^{n} E \chi\left(\frac{\eta_{t}}{\sigma}\right)+A_{n} \rightarrow 0
$$

Note that $\varepsilon_{t-1}$ and $\eta_{t}$ are independent; by $(2.8)$ and $E\left(\varepsilon_{t}\right)=0$, we have

$$
E\left(\frac{\partial Q}{\partial a}\right)=-\sum_{t=1}^{n} E\left(\psi\left(\frac{\eta_{t}}{\sigma}\right) \varepsilon_{t-1}\right)=-\sum_{t=1}^{n} E\left(\psi\left(\frac{\eta_{t}}{\sigma}\right)\right) E\left(\varepsilon_{t-1}\right)=0
$$

Hence, from (4.4)-(4.6),

$$
E\left(S_{n}(\theta)\right)=\left(0,-\sum_{t=1}^{n} E \chi\left(\frac{\eta_{t}}{\sigma}\right)+A_{n}, 0\right) \rightarrow 0
$$

By (2.6), we have

$$
\begin{aligned}
\operatorname{Var}\left(\frac{\partial Q}{\partial \beta^{T}}\right) & =\operatorname{Var}\left(\psi\left(\frac{\eta_{t}}{\sigma}\right)\right) \sum_{t=1}^{n}\left(\left(X_{t}-a X_{t-1}\right)\left(X_{t}-a X_{t-1}\right)^{T}+\left(1+a^{2}\right) \sigma_{\zeta}^{2} I_{d}\right) \\
& =E\left(\psi\left(\frac{\eta_{1}}{\sigma}\right)\right)^{2}\left(X_{n}(a)+n\left(1+a^{2}\right) \sigma_{\zeta}^{2} I_{d}\right) .
\end{aligned}
$$

By (2.7), we have

$$
\operatorname{Var}\left(\frac{\partial Q}{\partial \sigma}\right)=\sum_{t=1}^{n} \operatorname{Var}\left(\chi\left(\frac{\eta_{t}}{\sigma}\right)\right)=n \operatorname{Var}\left(\chi\left(\frac{\eta_{1}}{\sigma}\right)\right)
$$

Note that $\left\{\psi\left(\frac{\eta_{t}}{\sigma}\right) \varepsilon_{t-1}, H_{t}\right\}$ is a martingale difference sequence with

$$
\operatorname{Var}\left(\psi\left(\frac{\eta_{t}}{\sigma}\right) \varepsilon_{t-1}\right)=E\left(\psi\left(\frac{\eta_{t}}{\sigma}\right)\right)^{2} E \varepsilon_{t-1}^{2}
$$

so we have

$$
\begin{aligned}
\operatorname{Var}\left(\frac{\partial Q}{\partial a}\right) & =\sum_{t=1}^{n} \operatorname{Var}\left(\psi\left(\frac{\eta_{t}}{\sigma}\right) \varepsilon_{t-1}\right) \\
& =\sum_{t=1}^{n} E\left(\psi\left(\frac{\eta_{t}}{\sigma}\right)\right)^{2} E \varepsilon_{t-1}^{2}=E\left(\psi\left(\frac{\eta_{1}}{\sigma}\right)\right)^{2} \Delta_{n}(a, \sigma) .
\end{aligned}
$$

By (2.6) and (2.7), we have

$$
\begin{aligned}
\operatorname{Cov}\left(\frac{\partial Q}{\partial \beta^{T}}, \frac{\partial Q}{\partial \sigma}\right)= & E\left(\sum_{t=1}^{n} \psi\left(\frac{\eta_{t}}{\sigma}\right)\left(X_{t}-a X_{t-1}-\left(\zeta_{t}-a \zeta_{t-1}\right)\right)^{T}\right. \\
& \left.\cdot \sum_{t=1}^{n}\left(\chi\left(\frac{\eta_{t}}{\sigma}\right)-E \chi\left(\frac{\eta_{t}}{\sigma}\right)\right)\right)
\end{aligned}
$$




$$
\begin{aligned}
& =\sum_{t=1}^{n} E\left(\psi\left(\frac{\eta_{t}}{\sigma}\right)\left(\chi\left(\frac{\eta_{t}}{\sigma}\right)-E \chi\left(\frac{\eta_{t}}{\sigma}\right)\right)\right)\left(X_{t}-a X_{t-1}\right)^{T} \\
& =E\left(\psi\left(\frac{\eta_{1}}{\sigma}\right) \chi\left(\frac{\eta_{1}}{\sigma}\right)\right) \sum_{t=1}^{n}\left(X_{t}-a X_{t-1}\right)^{T} .
\end{aligned}
$$

By (2.6), (2.8), and noting that $\zeta_{t}, \varepsilon_{t-1}$, and $\eta_{t}$ are independent, we have

$$
\begin{aligned}
\operatorname{Cov}\left(\frac{\partial Q}{\partial \beta^{T}}, \frac{\partial Q}{\partial a}\right)= & E\left(\frac{\partial Q}{\partial \beta^{T}}, \frac{\partial Q}{\partial a}\right) \\
= & E\left(\sum_{t=1}^{n} \psi\left(\frac{\eta_{t}}{\sigma}\right)\left(X_{t}-a X_{t-1}-\left(\zeta_{t}-a \zeta_{t-1}\right)\right)^{T} \sum_{t=1}^{n} \psi\left(\frac{\eta_{t}}{\sigma}\right) \varepsilon_{t-1}\right) \\
= & \sum_{t=1}^{n} E\left(\psi^{2}\left(\frac{\eta_{t}}{\sigma}\right) \varepsilon_{t-1}\right)\left(X_{t}-a X_{t-1}-\left(\zeta_{t}-a \zeta_{t-1}\right)\right)^{T} \\
& +2 \sum_{t>k}^{n} E\left(\psi\left(\frac{\eta_{t}}{\sigma}\right) \psi\left(\frac{\eta_{k}}{\sigma}\right) \varepsilon_{k-1}\right) \\
= & \sum_{t=1}^{n} E \psi^{2}\left(\frac{\eta_{t}}{\sigma}\right) E \varepsilon_{t-1}\left(X_{t}-a X_{t-1}\right)^{T} \\
& +2 \sum_{t>k}^{n} E\left(\psi\left(\frac{\eta_{t}}{\sigma}\right) \psi\left(\frac{\eta_{k}}{\sigma}\right)\right)_{E \varepsilon_{k-1}} \\
= & 0 .
\end{aligned}
$$

By (2.7) and (2.8), we have

$$
\begin{aligned}
\operatorname{Cov}\left(\frac{\partial Q}{\partial a}, \frac{\partial Q}{\partial \sigma}\right)= & E\left(\sum_{t=1}^{n} \psi\left(\frac{\eta_{t}}{\sigma}\right) \varepsilon_{t-1} \sum_{t=1}^{n}\left(\chi\left(\frac{\eta_{t}}{\sigma}\right)-E \chi\left(\frac{\eta_{t}}{\sigma}\right)\right)\right) \\
= & \sum_{t=1}^{n} E\left(\psi\left(\frac{\eta_{t}}{\sigma}\right)\left(\chi\left(\frac{\eta_{t}}{\sigma}\right)-E \chi\left(\frac{\eta_{t}}{\sigma}\right)\right)\right) E \varepsilon_{t-1} \\
& +2 \sum_{t>k}^{n} E\left(\left(\chi\left(\frac{\eta_{t}}{\sigma}\right)-E \chi\left(\frac{\eta_{t}}{\sigma}\right)\right) \psi\left(\frac{\eta_{k}}{\sigma}\right)\right) E \varepsilon_{k-1} \\
= & 0 .
\end{aligned}
$$

Hence, (4.1) follows immediately from (4.7)-(4.12).

Similarly to the proof of $D_{n}(\theta)$, we easily prove that the matrix $\operatorname{Var}\left(S_{n}(\theta)\right)$ is positive definite. Thus, we complete the proof of Lemma 4.1.

Lemma 4.2 Assume that (A1) and (A4) hold. Then:

(1) for $|a|<1$, we have

$$
F_{n}(\theta)-D_{n}(\theta)=O_{p}\left(n^{\frac{1}{2}}\right), \quad n \rightarrow \infty
$$


(2) for $|a|=1$, we have

$$
F_{n}(\theta)-D_{n}(\theta)=\left(\begin{array}{ccc}
O_{p}\left(n^{\frac{1}{2}}\right) & O_{p}\left(n^{\frac{1}{2}}\right) & O_{p}(n) \\
* & O_{p}\left(n^{\frac{1}{2}}\right) & O_{p}\left(n^{\frac{1}{2}}\right) \\
* & * & O_{p}\left(n^{\frac{3}{2}}\right)
\end{array}\right)
$$

where the $*$ indicates that the elements are filled in by symmetry;

(3) for $|a|>1$, we have

$$
F_{n}(\theta)-D_{n}(\theta)=\left(\begin{array}{ccc}
O_{p}\left(n^{\frac{1}{2}}\right) & O_{p}\left(n^{\frac{1}{2}}\right) & O_{p}\left(a^{n}\right) \\
* & O_{p}\left(n^{\frac{1}{2}}\right) & O_{p}\left(n^{\frac{1}{2}}\right) \\
* & * & O_{p}\left(a^{2 n}\right)
\end{array}\right)
$$

Proof By (3.3) and (4.2), we obtain

$$
\begin{aligned}
n^{-1}\left\{X_{n}(a, \omega)-b X_{n}(a)-n b\left(1+a^{2}\right) \sigma_{\zeta}^{2} I_{d}\right\} \\
=\frac{1}{n \sigma} \sum_{t=1}^{n}\left\{\psi^{\prime}\left(\frac{\varepsilon_{t}-a \varepsilon_{t-1}}{\sigma}\right)-b\right\}\left(X_{t}-a X_{t-1}\right)\left(X_{t}-a X_{t-1}\right)^{T} \\
\quad+\frac{1}{n \sigma} \sum_{t=1}^{n}\left\{\psi^{\prime}\left(\frac{\varepsilon_{t}-a \varepsilon_{t-1}}{\sigma}\right) \zeta_{t} \zeta_{t}^{T}-b \sigma_{\zeta}^{2} I_{d}\right\} \\
\quad+\frac{a^{2}}{n \sigma} \sum_{t=1}^{n}\left\{\psi^{\prime}\left(\frac{\varepsilon_{t}-a \varepsilon_{t-1}}{\sigma}\right) \zeta_{t-1} \zeta_{t-1}^{T}-b \sigma_{\zeta}^{2} I_{d}\right\} \\
\quad-\frac{2 a}{n \sigma} \sum_{t=1}^{n} \psi^{\prime}\left(\frac{\varepsilon_{t}-a \varepsilon_{t-1}}{\sigma}\right) \zeta_{t} \zeta_{t-1}^{T}-\frac{2}{n \sigma} \sum_{t=1}^{n} \psi^{\prime}\left(\frac{\varepsilon_{t}-a \varepsilon_{t-1}}{\sigma}\right)\left(X_{t}-a X_{t-1}\right) \zeta_{t}^{T} \\
\quad+\frac{2 a}{n \sigma} \sum_{t=1}^{n} \psi^{\prime}\left(\frac{\varepsilon_{t}-a \varepsilon_{t-1}}{\sigma}\right)\left(X_{t}-a X_{t-1}\right) \zeta_{t-1}^{T} \\
=U_{1}+U_{2}+U_{3}+U_{4}+U_{5}+U_{6} .
\end{aligned}
$$

Note that $\left\{\psi^{\prime}\left(\frac{\eta_{t}}{\sigma}\right), t=1,2, \ldots, n\right\}$ are i.i.d. random variables with finite variance $\operatorname{Var}\left(\psi^{\prime}\left(\frac{\eta_{t}}{\sigma}\right)\right)$, we have

$$
\begin{aligned}
\operatorname{Var}\left\{n^{-1} \sum_{t=1}^{n}\left(\psi^{\prime}\left(\frac{\eta_{t}}{\sigma}\right)-E \psi^{\prime}\left(\frac{\eta_{t}}{\sigma}\right)\right)\right\} & =n^{-2} \sum_{t=1}^{n} \operatorname{Var}\left(\psi^{\prime}\left(\frac{\eta_{t}}{\sigma}\right)\right) \\
& =n^{-1} \operatorname{Var}\left(\psi^{\prime}\left(\frac{\eta_{1}}{\sigma}\right)\right)=O\left(n^{-1}\right) .
\end{aligned}
$$

By the Chebyshev inequality and (4.17), we have

$$
\begin{aligned}
\left(U_{1}\right)_{i j} & =\frac{1}{n \sigma} \sum_{t=1}^{n}\left\{\psi^{\prime}\left(\frac{\eta_{t}}{\sigma}\right)-E \psi^{\prime}\left(\frac{\eta_{t}}{\sigma}\right)\right\}\left(X_{t}-a X_{t-1}\right)_{i}\left(X_{t}-a X_{t-1}\right)_{j}^{T} \\
& \leq \frac{1}{\sigma} \max \left\|X_{t}-a X_{t-1}\right\|^{2} \cdot n^{-1} \sum_{t=1}^{n}\left\{\psi^{\prime}\left(\frac{\eta_{t}}{\sigma}\right)-E \psi^{\prime}\left(\frac{\eta_{t}}{\sigma}\right)\right\} \\
& =O_{p}\left(n^{-\frac{1}{2}}\right) .
\end{aligned}
$$


Similarly, we obtain

$$
\left(U_{i}\right)_{i j}=O_{p}\left(n^{-\frac{1}{2}}\right)
$$

By (4.16), (4.18), and (4.19), we have

$$
X_{n}(\theta, \omega)-b X_{n}(a)-n b\left(1+a^{2}\right) \sigma_{\zeta}^{2} I_{d}=O_{p}\left(n^{\frac{1}{2}}\right) .
$$

By (2.12) and (4.2), we easily obtain

$$
\begin{aligned}
& \frac{\partial^{2} Q}{\partial \beta^{T} \partial \sigma}-\frac{c}{\sigma} \sum_{t=1}^{n}\left(X_{t}-a X_{t-1}\right)^{T} \\
& =\frac{1}{\sigma} \sum_{t=1}^{n}\left\{\psi^{\prime}\left(\frac{\eta_{t}}{\sigma}\right) \frac{\eta_{t}}{\sigma}-E\left(\psi^{\prime}\left(\frac{\eta_{t}}{\sigma}\right) \frac{\eta_{t}}{\sigma}\right)\right\}\left(X_{t}-a X_{t-1}\right)^{T} \\
& \quad-\frac{1}{\sigma} \sum_{t=1}^{n} \psi^{\prime}\left(\frac{\eta_{t}}{\sigma}\right) \frac{\eta_{t}}{\sigma}\left(\zeta_{t}-a \zeta_{t-1}\right)^{T} \\
& =O_{p}\left(n^{\frac{1}{2}}\right)
\end{aligned}
$$

By (3.5) and (4.2), we obtain

$$
\begin{aligned}
n^{-\frac{1}{2}}\left\{\frac{\partial^{2} Q}{\partial \beta^{T} \partial a}-0\right\}= & n^{-\frac{1}{2}} \sum_{t=1}^{n} \psi^{\prime}\left(\frac{\eta_{t}}{\sigma}\right) \frac{\varepsilon_{t-1}}{\sigma}\left(X_{t}-a X_{t-1}\right)^{T} \\
& -n^{-\frac{1}{2}} \sum_{t=1}^{n} \psi^{\prime}\left(\frac{\eta_{t}}{\sigma}\right) \frac{\varepsilon_{t-1}}{\sigma}\left(\zeta_{t}-a \zeta_{t-1}\right)^{T} \\
& +n^{-\frac{1}{2}} \sum_{t=1}^{n} \psi\left(\frac{\eta_{t}}{\sigma}\right) X_{t-1}^{T}-n^{-\frac{1}{2}} \sum_{t=1}^{n} \psi\left(\frac{\eta_{t}}{\sigma}\right) \zeta_{t-1}^{T} \\
= & n^{-\frac{1}{2}}\left(U_{1}+U_{2}+U_{3}+U_{4}\right) .
\end{aligned}
$$

Note that $\left\{\psi^{\prime}\left(\frac{\eta_{t}}{\sigma}\right) \frac{\varepsilon_{t-1}}{\sigma}, H_{t}\right\}$ is a martingale difference sequence with

$$
\operatorname{Var}\left(\psi^{\prime}\left(\frac{\eta_{t}}{\sigma}\right) \frac{\varepsilon_{t-1}}{\sigma}\right)=E\left(\psi^{\prime}\left(\frac{\eta_{t}}{\sigma}\right)\right)^{2} E\left(\frac{\varepsilon_{t-1}^{2}}{\sigma^{2}}\right)
$$

so we have

$$
\begin{aligned}
& \left(\operatorname{Var}\left\{n^{-1} \sum_{t=1}^{n} \psi^{\prime}\left(\frac{\eta_{t}}{\sigma}\right) \frac{\varepsilon_{t-1}}{\sigma}\left(X_{t}-a X_{t-1}\right)^{T}\right\}\right)_{i j} \\
& \quad=n^{-2} \sum_{t=1}^{n} \operatorname{Var}\left\{\psi^{\prime}\left(\frac{\eta_{t}}{\sigma}\right) \frac{\varepsilon_{t-1}}{\sigma}\right\}\left(X_{t}-a X_{t-1}\right)_{i}\left(X_{t}-a X_{t-1}\right)_{j}^{T} \\
& \leq \frac{1}{\sigma^{2}} \max \left\|X_{t}-a X_{t-1}\right\|^{2} E\left(\psi^{\prime}\left(\frac{\eta_{1}}{\sigma}\right)\right)^{2} n^{-2} \sum_{t=1}^{n} E\left(\varepsilon_{t-1}^{2}\right) \\
& \quad=O\left(\Delta_{n}(a, \sigma) n^{-2}\right) .
\end{aligned}
$$


By the Chebyshev inequality and (4.23), we have

$$
\left(U_{1}\right)_{i}=O_{p}\left(\Delta_{n}^{\frac{1}{2}}(a, \sigma)\right)
$$

Similarly, we have

$$
\left(U_{2}\right)_{i}=O_{p}\left(\Delta_{n}^{\frac{1}{2}}(a, \sigma)\right)
$$

It is easy to show that

$$
\left(U_{3}\right)_{i}=O_{p}\left(n^{\frac{1}{2}}\right), \quad U_{4}=O_{p}\left(n^{\frac{1}{2}}\right) .
$$

By (4.22) and (4.24)-(4.26), we have

$$
\frac{\partial^{2} Q}{\partial \beta^{T} \partial a}-0=O_{p}\left(\Delta_{n}^{\frac{1}{2}}(a, \sigma)\right) .
$$

By (3.6) and (4.2), we obtain

$$
\begin{aligned}
\frac{\partial^{2} Q}{\partial \sigma^{2}}-\frac{n d}{\sigma} & =\frac{1}{\sigma}\left\{\sum_{t=1}^{n} \psi^{\prime}\left(\frac{\eta_{t}}{\sigma}\right) \eta_{t}^{2}-n d\right\}=\frac{1}{\sigma} \sum_{t=1}^{n}\left\{\psi^{\prime}\left(\frac{\eta_{t}}{\sigma}\right) \eta_{t}^{2}-E\left(\psi^{\prime}\left(\frac{\eta_{t}}{\sigma}\right) \eta_{t}^{2}\right)\right\} \\
& =O_{p}\left(n^{\frac{1}{2}}\right) .
\end{aligned}
$$

Note that $\left\{\psi^{\prime}\left(\frac{\eta_{t}}{\sigma}\right) \eta_{t} \varepsilon_{t-1}, H_{t}\right\}$ is a martingale difference sequence with $\operatorname{Var}\left(\psi^{\prime}\left(\frac{\eta_{t}}{\sigma}\right) \eta_{t} \varepsilon_{t-1}\right)=$ $\operatorname{Var}\left(\psi^{\prime}\left(\frac{\eta_{t}}{\sigma}\right) \eta_{t}\right) \operatorname{Var}\left(\varepsilon_{t-1}\right)$, and by (3.7) and (4.2), we obtain

$$
\frac{\partial^{2} Q}{\partial \sigma \partial a}-0=\frac{1}{\sigma^{2}} \sum_{t=1}^{n} \psi^{\prime}\left(\frac{\eta_{t}}{\sigma}\right) \eta_{t} \varepsilon_{t-1}=O_{p}\left(n^{\frac{1}{2}}\right) .
$$

By (3.8) and (4.2), we obtain

$$
\begin{aligned}
n^{-1}\left\{\frac{\partial^{2} Q}{\partial a^{2}}-\frac{b}{\sigma_{0}} \Delta_{n}(a, \sigma)\right\}= & \frac{1}{n \sigma}\left\{\sum_{t=1}^{n} \psi^{\prime}\left(\frac{\eta_{t}}{\sigma}\right) \varepsilon_{t-1}^{2}-b \Delta_{n}(a, \sigma)\right\} \\
= & \frac{1}{n \sigma} \sum_{t=1}^{n}\left\{\psi^{\prime}\left(\frac{\eta_{t}}{\sigma}\right)-E\left(\psi^{\prime}\left(\frac{\eta_{t}}{\sigma}\right)\right)\right\} \varepsilon_{t-1}^{2} \\
& +\frac{b}{\Delta_{n}(a, \sigma) \sigma} \sum_{t=1}^{n}\left(\varepsilon_{t-1}^{2}-E\left(\varepsilon_{t-1}^{2}\right)\right) \\
= & T_{1}+T_{2} .
\end{aligned}
$$

Since we easily prove that $\left\{\psi^{\prime}\left(\frac{\eta_{t}}{\sigma}\right)-E\left(\psi^{\prime}\left(\frac{\eta_{t}}{\sigma}\right)\right) \varepsilon_{t-1}^{2}, H_{t}\right\}$ is a martingale difference sequence,

$$
\begin{aligned}
\operatorname{Var}\left(T_{1}\right) & =\frac{1}{(n \sigma)^{2}} \sum_{t=1}^{n} E\left\{\psi^{\prime}\left(\frac{\eta_{t}}{\sigma}\right)-E\left(\psi^{\prime}\left(\frac{\eta_{t}}{\sigma}\right)\right)\right\}^{2} E\left(\varepsilon_{t-1}^{4}\right) \\
& =\frac{1}{(n \sigma)^{2}} \operatorname{Var}\left\{\psi^{\prime}\left(\frac{\eta_{1}}{\sigma}\right)\right\} \sum_{t=1}^{n} E\left(\varepsilon_{t-1}^{4}\right) .
\end{aligned}
$$


By (2.1), we obtain

$$
\begin{aligned}
E\left(\varepsilon_{t-1}^{4}\right) & \leq \sum_{j=1}^{t-1} a^{4(t-1-j)} E \eta_{j}^{4}+\sigma^{4} \sum_{j=1}^{t-1} a^{2(t-1-j)} \sum_{k=1}^{t-1} a^{2(t-1-k)} \\
& = \begin{cases}E \eta_{1}^{4} \frac{a^{4(t-2)}\left(1-a^{-4(t-1)}\right)}{1-a^{-4}}+3 \sigma^{4}\left(\frac{a^{2(t-2)}\left(1-a^{-2(t-1)}\right)}{1-a^{-2}}\right)^{2}, & \text { if }|a| \neq 1, \\
E \eta_{1}^{4}(t-1)+\sigma^{4}(t-1)^{2}, & \text { if }|a|=1 .\end{cases}
\end{aligned}
$$

Thus

$$
\begin{aligned}
& \sum_{t=1}^{n} E\left(\varepsilon_{t-1}^{4}\right) \\
& \quad \leq \begin{cases}E \eta_{1}^{4}\left(\frac{a^{4 n}-1}{\left(1-a^{-4}\right)^{2}}-\frac{n}{a^{4}-1}\right)+\frac{3 \sigma^{4}}{\left(1-a^{-2}\right)^{2}}\left(\frac{a^{4 n}-1}{1-a^{-4}}-\frac{2\left(a^{2 n}-1\right)}{a^{-2}\left(1-a^{-2}\right)}+n a^{-4}\right), & |a| \neq 1, \\
\frac{1}{2} E \eta_{1}^{4} n(n-1)+\sigma^{4} \frac{(n-1) n(2 n-1)}{6}, & |a|=1 .\end{cases}
\end{aligned}
$$

That is,

$$
\sum_{t=1}^{n} E\left(\varepsilon_{t-1}^{4}\right)= \begin{cases}O(n), & \text { if }|a|<1 \\ O\left(a^{4 n}\right), & \text { if }|a|>1 \\ O\left(n^{3}\right), & \text { if }|a|=1\end{cases}
$$

By Chebyshev inequality and (4.31)-(4.34), we have

$$
T_{1}= \begin{cases}O_{p}\left(n^{-\frac{1}{2}}\right), & \text { if }|a|<1, \\ O_{p}\left(n^{-1} a^{2 n}\right), & \text { if }|a|>1, \\ O_{p}\left(n^{\frac{1}{2}}\right), & \text { if }|a|=1 .\end{cases}
$$

Similarly to the proof of (4.35), we easily obtain

$$
T_{2}= \begin{cases}O_{p}\left(n^{-\frac{1}{2}}\right), & \text { if }\left|a_{0}\right|<1, \\ O_{p}(1), & \text { if }\left|a_{0}\right|>1, \\ O_{p}\left(n^{-\frac{1}{2}}\right), & \text { if }\left|a_{0}\right|=1 .\end{cases}
$$

Hence, by (4.30), (4.35), and (4.36), we have

$$
\frac{\partial^{2} Q}{\partial a^{2}}-\frac{b}{\sigma} \Delta_{n}(\theta)= \begin{cases}O_{p}\left(n^{\frac{1}{2}}\right), & \text { if }|a|<1, \\ O_{p}\left(a^{2 n}\right), & \text { if }|a|>1, \\ O_{p}\left(n^{\frac{3}{2}}\right), & \text { if }|a|=1 .\end{cases}
$$

Thus Lemma 4.2 follows from (4.20), (4.21), (4.27)-(4.29), and (4.37).

Lemma 4.3 Assume that (A1), (A3), and (A4) hold, and $E \eta_{t}^{6}<\infty, E\left(\psi^{\prime \prime}\left(\frac{\eta_{t}}{\sigma}\right)\right)^{2}<\infty$ and $\theta \in \Theta$. Then, as $n \rightarrow \infty$ :

(1) for $|a|<1$, we have

$$
R_{n l}(\theta)=\frac{\partial}{\partial \theta_{l}} \frac{\partial^{2}}{\partial \theta^{T} \partial \theta} Q(\theta)=\frac{\partial}{\partial \theta_{l}} F_{n}(\theta)=O_{p}\left(n^{\frac{1}{2}}\right), \quad l=1,2, \ldots, d+2
$$


(2) for $|a|=1$, we have

$$
R_{n l}(\theta)=\left(\begin{array}{ccc}
\left(\begin{array}{ccc}
O_{p}\left(n^{\frac{1}{2}}\right) & O_{p}\left(n^{\frac{1}{2}}\right) & O_{p}(n) \\
* & O_{p}\left(n^{\frac{1}{2}}\right) & O_{p}(n) \\
* & * & O_{p}(n)
\end{array}\right)_{(d+2) \times(d+2)} \\
\left(\begin{array}{ccc}
O_{p}\left(n^{\frac{1}{2}}\right) & O_{p}\left(n^{\frac{1}{2}}\right) & O_{p}(n) \\
* & O_{p}\left(n^{\frac{1}{2}}\right) & O_{p}(n) \\
* & * & O_{p}(n)
\end{array}\right)_{(d+2) \times(d+2)} \\
\left(\begin{array}{ccc}
O_{p}\left(n^{\frac{1}{2}}\right) & O_{p}\left(n^{\frac{1}{2}}\right) & O_{p}(n) \\
* & O_{p}\left(n^{\frac{1}{2}}\right) & O_{p}(n) \\
* & * & O_{p}\left(n^{\frac{3}{2}}\right)
\end{array}\right)_{(d+2) \times(d+2)}{ }_{\left(n^{\frac{3}{2}}\right)} O_{p}\left(n^{\frac{3}{2}}\right) & O_{p}\left(n^{\frac{3}{2}}\right) \\
* & O_{p}\left(n^{\frac{3}{2}}\right) & O_{p}\left(n^{\frac{3}{2}}\right) \\
* & * & O_{p}\left(n^{2}\right)
\end{array}\right)_{(d+2) \times(d+2)}
$$

(3) for $|a|>1$, we have

$$
R_{n l}(\theta)=\left(\begin{array}{ccc}
\left(\begin{array}{ccc}
O_{p}\left(n^{\frac{1}{2}}\right) & O_{p}\left(n^{\frac{1}{2}}\right) & O_{p}\left(a^{n}\right) \\
* & O_{p}\left(n^{\frac{1}{2}}\right) & O_{p}\left(a^{n}\right) \\
* & * & O_{p}\left(a^{n}\right)
\end{array}\right)_{(d+2) \times(d+2)} \\
\left(\begin{array}{ccc}
O_{p}\left(n^{\frac{1}{2}}\right) & O_{p}\left(n^{\frac{1}{2}}\right) & O_{p}\left(a^{n}\right) \\
* & O_{p}\left(n^{\frac{1}{2}}\right) & O_{p}\left(a^{n}\right) \\
* & * & O_{p}\left(a^{n}\right)
\end{array}\right)_{(d+2) \times(d+2)} \\
\left.\left(\begin{array}{ccc}
O_{p}\left(n^{\frac{1}{2}}\right) & O_{p}\left(n^{\frac{1}{2}}\right) & O_{p}\left(a^{n}\right) \\
* & O_{p}\left(n^{\frac{1}{2}}\right) & O_{p}\left(a^{n}\right) \\
* & * & O_{p}\left(a^{2 n}\right)
\end{array}\right)_{(d+2) \times(d+2)}\right)_{\left(a^{n}\right)} O_{p}\left(a^{n}\right) & O_{p}\left(a^{2 n}\right) \\
* & O_{p}\left(a^{n}\right) & O_{p}\left(a^{2 n}\right) \\
* & * & O_{p}\left(a^{3 n}\right)
\end{array}\right)_{(d+2) \times(d+2)}
$$

Proof Let

$$
\frac{\partial}{\partial \theta_{l}} F_{n}(\theta)=\left(\begin{array}{ccc}
\frac{\partial^{3} Q}{\partial \theta_{l} \partial \beta^{T} \partial \beta} & \frac{\partial^{3} Q}{\partial \theta_{l} \partial \beta^{T} \partial \sigma} & \frac{\partial^{3} Q}{\partial \theta_{l} \partial \beta^{T} \partial a} \\
* & \frac{\partial^{3} Q}{\partial \theta_{l} \partial \sigma^{2}} & \frac{\partial^{3} Q}{\partial \theta_{l} \partial \sigma \partial a} \\
* & * & \frac{\partial^{3} Q}{\partial \theta_{l} \partial a^{2}}
\end{array}\right) .
$$

Case 1. $l=1,2, \ldots, d$.

$$
\begin{aligned}
\frac{\partial^{3} Q}{\partial \theta_{l} \partial \beta^{T} \partial \beta}= & -\frac{1}{\sigma^{2}} \sum_{t=1}^{n} \psi^{\prime \prime}\left(\frac{\varepsilon_{t}-a \varepsilon_{t-1}}{\sigma}\right)\left(X_{t l}-a X_{t-1, l}-\left(\zeta_{t l}-a \zeta_{t-1, l}\right)\right) \\
& \cdot\left(X_{t}-a X_{t-1}-\left(\zeta_{t}-a \zeta_{t-1}\right)\right)\left(X_{t}-a X_{t-1}-\left(\zeta_{t}-a \zeta_{t-1}\right)\right)^{T}
\end{aligned}
$$




$$
\begin{aligned}
& \frac{\partial^{3} Q}{\partial \theta_{l} \partial \beta^{T} \partial \sigma}=-\frac{1}{\sigma^{2}} \sum_{t=1}^{n}\left\{\psi^{\prime}\left(\frac{\varepsilon_{t}-a \varepsilon_{t-1}}{\sigma}\right)+\psi^{\prime \prime}\left(\frac{\varepsilon_{t}-a \varepsilon_{t-1}}{\sigma}\right) \frac{\varepsilon_{t}-a \varepsilon_{t-1}}{\sigma}\right\} \\
& \cdot\left(X_{t l}-a X_{t-1, l}-\left(\zeta_{t l}-a \zeta_{t-1, l}\right)\right)\left(X_{t}-a X_{t-1}-\left(\zeta_{t}-a \zeta_{t-1}\right)\right)^{T} \\
& \frac{\partial^{3} Q}{\partial \theta_{l} \partial \beta^{T} \partial a}=-\frac{1}{\sigma} \sum_{t=1}^{n} \psi^{\prime \prime}\left(\frac{\varepsilon_{t}-a \varepsilon_{t-1}}{\sigma}\right) \frac{\varepsilon_{t-1}}{\sigma} \\
& \cdot\left(X_{t l}-a X_{t-1, l}-\left(\zeta_{t l}-a \zeta_{t-1, l}\right)\right)\left(X_{t}-a X_{t-1}-\left(\zeta_{t}-a \zeta_{t-1}\right)\right)^{T} \\
& -\frac{1}{\sigma} \sum_{t=1}^{n} \psi^{\prime}\left(\frac{\varepsilon_{t}-a \varepsilon_{t-1}}{\sigma}\right)\left\{\left(X_{t-1, l}-\zeta_{t-1, l}\right)\left(X_{t}-a X_{t-1}-\left(\zeta_{t}-a \zeta_{t-1}\right)\right)^{T}\right. \\
& \left.+\left(X_{t l}-a X_{t-1, l}-\left(\zeta_{t l}-a \zeta_{t-1, l}\right)\right)\left(X_{t-1}-\zeta_{t-1}\right)^{T}\right\}, \\
& \frac{\partial^{3} Q}{\partial \theta_{l} \partial \sigma^{2}}=-\frac{1}{\sigma^{2}} \sum_{t=1}^{n} \psi^{\prime \prime}\left(\frac{\varepsilon_{t}-a \varepsilon_{t-1}}{\sigma}\right)\left(X_{t l}-a X_{t-1, l}-\left(\zeta_{t l}-a \zeta_{t-1, l}\right)\right)\left(\varepsilon_{t}-a \varepsilon_{t-1}\right)^{2} \\
& -\frac{2}{\sigma} \sum_{t=1}^{n} \psi^{\prime}\left(\frac{\varepsilon_{t}-a \varepsilon_{t-1}}{\sigma}\right)\left(X_{t l}-a X_{t-1, l}-\left(\zeta_{t l}-a \zeta_{t-1, l}\right)\right)\left(\varepsilon_{t}-a \varepsilon_{t-1}\right) \\
& \frac{\partial^{3} Q}{\partial \theta_{l} \partial \sigma \partial a}=-\frac{1}{\sigma^{2}} \sum_{t=1}^{n} \psi^{\prime \prime}\left(\frac{\varepsilon_{t}-a \varepsilon_{t-1}}{\sigma}\right) \frac{\varepsilon_{t}-a \varepsilon_{t-1}}{\sigma} \varepsilon_{t-1}\left(X_{t l}-a X_{t-1, l}-\left(\zeta_{t l}-a \zeta_{t-1, l}\right)\right) \\
& -\frac{1}{\sigma^{2}} \sum_{t=1}^{n} \psi^{\prime}\left(\frac{\varepsilon_{t}-a \varepsilon_{t-1}}{\sigma}\right) \varepsilon_{t-1}\left(X_{t l}-a X_{t-1, l}-\left(\zeta_{t l}-a \zeta_{t-1, l}\right)\right) \\
& -\frac{1}{\sigma^{2}} \sum_{t=1}^{n} \psi^{\prime}\left(\frac{\varepsilon_{t}-a \varepsilon_{t-1}}{\sigma}\right)\left(\varepsilon_{t}-a \varepsilon_{t-1}\right)\left(X_{t-1, l}-\zeta_{t-1, l}\right)
\end{aligned}
$$

and

$$
\begin{aligned}
\frac{\partial^{3} Q}{\partial \theta_{l} \partial a^{2}}= & -\frac{1}{\sigma^{2}} \sum_{t=1}^{n} \psi^{\prime \prime}\left(\frac{\varepsilon_{t}-a \varepsilon_{t-1}}{\sigma}\right) \varepsilon_{t-1}^{2}\left(X_{t l}-a X_{t-1, l}-\left(\zeta_{t l}-a \zeta_{t-1, l}\right)\right) \\
& -\frac{2}{\sigma} \sum_{t=1}^{n} \psi^{\prime}\left(\frac{\varepsilon_{t}-a \varepsilon_{t-1}}{\sigma}\right) \varepsilon_{t-1}\left(X_{t-1, l}-\zeta_{t-1, l}\right) .
\end{aligned}
$$

Case $2 . l=d+1$

$$
\begin{aligned}
\frac{\partial^{3} Q}{\partial \sigma \partial \beta^{T} \partial \beta}= & -\frac{1}{\sigma^{2}} \sum_{t=1}^{n}\left(\psi^{\prime \prime}\left(\frac{\varepsilon_{t}-a \varepsilon_{t-1}}{\sigma}\right) \frac{\varepsilon_{t}-a \varepsilon_{t-1}}{\sigma}+\psi^{\prime}\left(\frac{\varepsilon_{t}-a \varepsilon_{t-1}}{\sigma}\right)\right) \\
& \cdot\left(X_{t}-a X_{t-1}-\left(\zeta_{t}-a \zeta_{t-1}\right)\right)\left(X_{t}-a X_{t-1}-\left(\zeta_{t}-a \zeta_{t-1}\right)\right)^{T} \\
\frac{\partial^{3} Q}{\partial \sigma \partial \beta^{T} \partial \sigma}= & -\frac{1}{\sigma^{3}} \sum_{t=1}^{n}\left\{2 \psi^{\prime}\left(\frac{\varepsilon_{t}-a \varepsilon_{t-1}}{\sigma}\right)+\psi^{\prime \prime}\left(\frac{\varepsilon_{t}-a \varepsilon_{t-1}}{\sigma}\right) \frac{\varepsilon_{t}-a \varepsilon_{t-1}}{\sigma}\right\} \\
& \cdot\left(\varepsilon_{t}-a \varepsilon_{t-1}\right)\left(X_{t}-a X_{t-1}-\left(\zeta_{t}-a \zeta_{t-1}\right)\right)^{T} \\
\frac{\partial^{3} Q}{\partial \sigma \partial \beta^{T} \partial a}= & -\frac{1}{\sigma} \sum_{t=1}^{n} \psi^{\prime \prime}\left(\frac{\varepsilon_{t}-a \varepsilon_{t-1}}{\sigma}\right) \frac{\varepsilon_{t}-a \varepsilon_{t-1}}{\sigma} \frac{\varepsilon_{t-1}}{\sigma}\left(X_{t}-a X_{t-1}-\left(\zeta_{t}-a \zeta_{t-1}\right)\right)^{T}
\end{aligned}
$$




$$
\begin{aligned}
& -\frac{1}{\sigma} \sum_{t=1}^{n} \psi^{\prime}\left(\frac{\varepsilon_{t}-a \varepsilon_{t-1}}{\sigma}\right) \\
& \cdot\left(\frac{\varepsilon_{t-1}}{\sigma}\left(X_{t}-a X_{t-1}-\left(\zeta_{t}-a \zeta_{t-1}\right)\right)^{T}+\frac{\varepsilon_{t}-a \varepsilon_{t-1}}{\sigma}\right), \\
\frac{\partial^{3} Q}{\partial \sigma^{3}}= & \frac{1}{\sigma^{3}} \sum_{t=1}^{n}\left(2 \psi^{\prime}\left(\frac{\varepsilon_{t}-a \varepsilon_{t-1}}{\sigma}\right)\right. \\
& \left.+\psi^{\prime \prime}\left(\frac{\varepsilon_{t}-a \varepsilon_{t-1}}{\sigma}\right) \frac{\varepsilon_{t}-a \varepsilon_{t-1}}{\sigma}\right)\left(\varepsilon_{t}-a \varepsilon_{t-1}\right)^{2}, \\
\frac{\partial^{3} Q}{\partial \sigma^{2} \partial a}= & -\frac{1}{\sigma^{4}} \sum_{t=1}^{n} \psi^{\prime \prime}\left(\frac{\varepsilon_{t}-a \varepsilon_{t-1}}{\sigma}\right)\left(\varepsilon_{t}-a \varepsilon_{t-1}\right)^{2} \varepsilon_{t-1} \\
& -\frac{2}{\sigma^{3}} \sum_{t=1}^{n} \psi^{\prime}\left(\frac{\varepsilon_{t}-a \varepsilon_{t-1}}{\sigma}\right)\left(\varepsilon_{t}-a \varepsilon_{t-1}\right) \varepsilon_{t-1},
\end{aligned}
$$

and

$$
\frac{\partial^{3} Q}{\partial \sigma \partial a^{2}}=-\frac{1}{\sigma^{2}} \sum_{t=1}^{n}\left(\psi^{\prime \prime}\left(\frac{\varepsilon_{t}-a \varepsilon_{t-1}}{\sigma}\right) \frac{\varepsilon_{t}-a \varepsilon_{t-1}}{\sigma}+\psi^{\prime}\left(\frac{\varepsilon_{t}-a \varepsilon_{t-1}}{\sigma}\right)\right) \varepsilon_{t-1}^{2} .
$$

Case 3. $l=d+2$.

$$
\begin{aligned}
& \frac{\partial^{3} Q}{\partial a \partial \beta^{T} \partial \beta}=-\frac{1}{\sigma} \sum_{t=1}^{n} \psi^{\prime}\left(\frac{\varepsilon_{t}-a \varepsilon_{t-1}}{\sigma}\right) \\
& \cdot \frac{\varepsilon_{t-1}}{\sigma}\left(X_{t}-a X_{t-1}-\left(\zeta_{t}-a \zeta_{t-1}\right)\right)\left(X_{t}-a X_{t-1}-\left(\zeta_{t}-a \zeta_{t-1}\right)\right)^{T} \\
& -\frac{1}{\sigma} \sum_{t=1}^{n} \psi^{\prime}\left(\frac{\varepsilon_{t}-a \varepsilon_{t-1}}{\sigma}\right)\left(X_{t-1}-\zeta_{t-1}\right)\left(X_{t}-a X_{t-1}-\left(\zeta_{t}-a \zeta_{t-1}\right)\right)^{T} \\
& -\frac{1}{\sigma} \sum_{t=1}^{n} \psi^{\prime}\left(\frac{\varepsilon_{t}-a \varepsilon_{t-1}}{\sigma}\right)\left(X_{t}-a X_{t-1}-\left(\zeta_{t}-a \zeta_{t-1}\right)\right)\left(X_{t-1}-\zeta_{t-1}\right)^{T}, \\
& \frac{\partial^{3} Q}{\partial a \partial \beta^{T} \partial \sigma}=-\frac{1}{\sigma^{2}} \sum_{t=1}^{n} \psi^{\prime}\left(\frac{\varepsilon_{t}-a \varepsilon_{t-1}}{\sigma}\right) \\
& \cdot\left(\left(\varepsilon_{t}-a \varepsilon_{t-1}\right)\left(X_{t-1}-\zeta_{t-1}\right)^{T}+\varepsilon_{t-1}\left(X_{t}-a X_{t-1}-\left(\zeta_{t}-a \zeta_{t-1}\right)\right)^{T}\right) \\
& -\frac{1}{\sigma^{2}} \sum_{t=1}^{n} \psi^{\prime \prime}\left(\frac{\varepsilon_{t}-a \varepsilon_{t-1}}{\sigma}\right) \frac{\varepsilon_{t-1}}{\sigma}\left(\varepsilon_{t}-a \varepsilon_{t-1}\right) \\
& \cdot\left(X_{t}-a X_{t-1}-\left(\zeta_{t}-a \zeta_{t-1}\right)\right)^{T} \\
& \frac{\partial^{3} Q}{\partial a \partial \beta^{T} \partial a}=-\frac{1}{\sigma^{2}} \sum_{t=1}^{n} \psi^{\prime \prime}\left(\frac{\varepsilon_{t}-a \varepsilon_{t-1}}{\sigma}\right) \varepsilon_{t-1}^{2}\left(X_{t}-a X_{t-1}-\left(\zeta_{t}-a \zeta_{t-1}\right)\right)^{T}, \\
& \frac{\partial^{3} Q}{\partial a \partial \sigma^{2}}=-\frac{1}{\sigma^{2}} \sum_{t=1}^{n} \psi^{\prime \prime}\left(\frac{\varepsilon_{t}-a \varepsilon_{t-1}}{\sigma}\right) \varepsilon_{t-1}\left(\varepsilon_{t}-a \varepsilon_{t-1}\right)^{2} \\
& -\frac{2}{\sigma} \sum_{t=1}^{n} \psi^{\prime}\left(\frac{\varepsilon_{t}-a \varepsilon_{t-1}}{\sigma}\right)\left(\varepsilon_{t}-a \varepsilon_{t-1}\right) \varepsilon_{t-1},
\end{aligned}
$$




$$
\frac{\partial^{3} Q}{\partial a \partial \sigma \partial a}=-\frac{1}{\sigma^{2}} \sum_{t=1}^{n}\left(\psi^{\prime \prime}\left(\frac{\varepsilon_{t}-a \varepsilon_{t-1}}{\sigma}\right) \frac{\varepsilon_{t}-a \varepsilon_{t-1}}{\sigma}+\psi^{\prime}\left(\frac{\varepsilon_{t}-a \varepsilon_{t-1}}{\sigma}\right)\right) \varepsilon_{t-1}^{2},
$$

and

$$
\frac{\partial^{3} Q}{\partial a^{3}}=-\frac{1}{\sigma^{2}} \sum_{t=1}^{n} \psi^{\prime \prime}\left(\frac{\varepsilon_{t}-a \varepsilon_{t-1}}{\sigma}\right) \varepsilon_{t-1}^{3}
$$

Similarly to the proof of Lemma 4.2, we easily obtain Lemma 4.3. Here we omit it.

Lemma 4.4 (Prakasa Rao [38]) If $\left\{X_{n}, n \geq 1\right\}$ are independent random variables with $E X_{n}=0$, and $s_{n}^{-(2+\delta)} \sum_{j=1}^{n} E\left|X_{j}\right|^{2+\delta} \rightarrow 0$ for some $\delta>0$, then

$$
s_{n}^{-1} \sum_{j=1}^{n} X_{j} \rightarrow N(0,1)
$$

where $s_{n}^{2}=\sum_{j=1}^{n} E X_{j}^{2}$.

Lemma 4.5 (Hall and Heyde [39]) Let $\left\{S_{n i}, F_{n i}, 1 \leq i \leq k_{n}, n \geq 1\right\}$ be a zero-mean, squareintegrable martingale array with differences $X_{n i}$, and let $\eta^{2}$ be an a.s. finite random variable. Suppose that $\sum_{i} E\left\{X_{n i}^{2} I\left(\left|X_{n i}\right|>\varepsilon\right) \mid F_{n, i-1}\right\} \rightarrow_{p} 0$, for all $\varepsilon \rightarrow 0$, and $\sum_{i} E\left\{X_{n i}^{2} \mid F_{n, i-1}\right\} \rightarrow_{p}$ $\eta^{2}$. Then

$$
S_{n k_{n}}=\sum_{i} X_{n i} \rightarrow_{D} Z
$$

where the r.v. $Z$ has characteristic function $E\left\{\exp \left(-\frac{1}{2} \eta^{2} t^{2}\right)\right\}$.

Proof of Theorem 3.1 Expanding $\frac{\partial}{\partial \theta} Q\left(\hat{\theta}_{n}\right)$ about $\theta$, we have

$$
\frac{\partial}{\partial \theta} Q\left(\hat{\theta}_{n}\right)=\frac{\partial}{\partial \theta} Q(\theta)+\left(\hat{\theta}_{n}-\theta\right) \frac{\partial^{2}}{\partial \theta^{T} \partial \theta} Q(\theta)+\frac{1}{2}\left[\tilde{R}_{n l}\left(\bar{\theta}, \hat{\theta}_{n}, \theta\right)\right]_{1 \leq l \leq d+2},
$$

where $\bar{\theta}=s \hat{\theta}_{n}+(1-s) \theta$ for some $0 \leq s \leq 1$ and

$$
\left[\tilde{R}_{n l}\left(\bar{\theta}, \hat{\theta}_{n}, \theta\right)\right]_{1 \leq l \leq d+2}=\left\{\left(\hat{\theta}_{n}-\theta\right) R_{n 1}(\bar{\theta})\left(\hat{\theta}_{n}-\theta\right)^{T}, \ldots,\left(\hat{\theta}_{n}-\theta\right) R_{n, d+2}(\bar{\theta})\left(\hat{\theta}_{n}-\theta\right)^{T}\right\}
$$

By (2.9)-(2.11) and (3.1), (3.2), we have

$$
0=S_{n}(\theta)+\left(\hat{\theta}_{n}-\theta\right) F_{n}(\theta)+\frac{1}{2}\left[\tilde{R}_{n l}\left(\bar{\theta}, \hat{\theta}_{n}, \theta\right)\right]_{1 \leq l \leq d+2} .
$$

By (4.58), we have

$$
\left(\hat{\theta}_{n}-\theta\right) D_{n}(\theta)=-S_{n}(\theta)-\left(\hat{\theta}_{n}-\theta\right)\left(D_{n}(\theta)-F_{n}(\theta)\right)-\frac{1}{2}\left[\tilde{R}_{n l}\left(\bar{\theta}, \hat{\theta}_{n}, \theta\right)\right]_{1 \leq l \leq d+2} .
$$


(1) $|a|<1$. By Lemma 4.3(1), Lemma 4.2(1), (2.9)-(2.11), and $\theta \in \Theta_{1}$, we have

$$
\begin{aligned}
n^{-\frac{1}{2}}\left(\hat{\theta}_{n}-\theta\right) D_{n}(\theta)= & -n^{-\frac{1}{2}} S_{n}(\theta)+o_{p}(1) \\
= & n^{-\frac{1}{2}}\left\{\sum_{t=1}^{n} \psi\left(\frac{\eta_{t}}{\sigma}\right)\left(X_{t}-a X_{t-1}-\left(\zeta_{t}-a \zeta_{t-1}\right)\right)^{T},\right. \\
& \left.\sum_{t=1}^{n} \chi\left(\frac{\eta_{t}}{\sigma}\right)-A_{n}, \sum_{t=1}^{n} \psi\left(\frac{\eta_{t}}{\sigma}\right) \varepsilon_{t-1}\right\}+o_{p}(1) \\
= & n^{-\frac{1}{2}}\left\{\sum_{t=1}^{n} \psi\left(\frac{\eta_{t}}{\sigma}\right)\left(X_{t}-a X_{t-1}-\left(\zeta_{t}-a \zeta_{t-1}\right)\right)^{T},\right. \\
& \left.\sum_{t=1}^{n}\left(\chi\left(\frac{\eta_{t}}{\sigma}\right)-A\right), \sum_{t=1}^{n} \psi\left(\frac{\eta_{t}}{\sigma}\right) \varepsilon_{t-1}\right\}+o_{p}(1) .
\end{aligned}
$$

Note that $\operatorname{Var}\left(S_{n}(\theta)\right)=O(n)$, so we have

$$
\begin{aligned}
\left(\hat{\theta}_{n}-\theta\right) D_{n}(\theta) \operatorname{Var}^{-\frac{1}{2}}\left(S_{n}(\theta)\right)= & -S_{n}(\theta) \operatorname{Var}^{-\frac{1}{2}}\left(S_{n}(\theta)\right)+o_{p}(1) \\
= & \left\{-\left[S_{n}(\theta)\right]_{\beta, \sigma}\left[\operatorname{Var}^{-\frac{1}{2}}\left(S_{n}(\theta)\right)\right]_{\beta, \sigma},\right. \\
& \left.\sum_{t=1}^{n} \psi\left(\frac{\eta_{t}}{\sigma}\right) \varepsilon_{t-1}\left(E \psi^{2}\left(\frac{\eta_{1}}{\sigma}\right) \Delta_{n}(a, \sigma)\right)^{-\frac{1}{2}}\right\} \\
& +o_{p}(1) .
\end{aligned}
$$

By Lemma 2 of Silvapullé [25], we easily obtain

$$
-\left[S_{n}(\theta)\right]_{\beta, \sigma}\left[\operatorname{Var}^{-\frac{1}{2}}\left(S_{n}(\theta)\right)\right]_{\beta, \sigma} \rightarrow_{D} N\left(0, I_{d+1}\right), \quad n \rightarrow \infty .
$$

In the following, we will prove that

$$
\sum_{t=1}^{n} \psi\left(\frac{\eta_{t}}{\sigma}\right) \varepsilon_{t-1}\left(E \psi^{2}\left(\frac{\eta_{1}}{\sigma}\right) \Delta_{n}(a, \sigma)\right)^{-\frac{1}{2}} \rightarrow N(0,1), \quad n \rightarrow \infty .
$$

Note that $\left\{\psi\left(\frac{\eta_{t}}{\sigma}\right) \varepsilon_{t-1}\left(E \psi^{2}\left(\frac{\eta_{1}}{\sigma}\right) \Delta_{n}(a, \sigma)\right)^{-\frac{1}{2}}, H_{t}\right\}$ is a martingale differences sequence, so we will verify the Lindeberg conditions for their convergence to normality.

From (1.2), we have

$$
\begin{aligned}
\left(1-a^{2}\right) \sum_{t=2}^{n} \varepsilon_{t-1}^{2}+\varepsilon_{n}^{2}-\varepsilon_{1}^{2} & =\sum_{t=2}^{n}\left(\varepsilon_{t}^{2}-a^{2} \varepsilon_{t-1}^{2}\right) \\
& =\sum_{t=2}^{n}\left(\varepsilon_{t}-a \varepsilon_{t-1}\right)\left(\varepsilon_{t}+a \varepsilon_{t-1}\right) \\
& =\sum_{t=2}^{n} \eta_{t}\left(\eta_{t}+2 a \varepsilon_{t-1}\right) \\
& =\sum_{t=2}^{n} \eta_{t}^{2}+2 a \sum_{t=2}^{n} \eta_{t} \varepsilon_{t-1} .
\end{aligned}
$$


By $\operatorname{Var}\left(\sum_{t=2}^{n} \eta_{t} \varepsilon_{t-1}\right)=\sigma^{2} \Delta_{n}(a, \sigma)=\sigma^{2} n$ and Chebyshev inequality, we have

$$
\sum_{t=2}^{n} \eta_{t} \varepsilon_{t-1}=O_{p}\left(n^{\frac{1}{2}}\right)
$$

Obviously, $n^{-1} \sum_{t=2}^{n} \eta_{t}^{2} \rightarrow_{p} E \eta_{1}^{2}$. By (4.46) and $\max _{1 \leq t \leq n} \frac{\varepsilon_{t}^{2}}{n}=o_{p}(1)$, we have

$$
\sum_{t=2}^{n} \varepsilon_{t-1}^{2}=O_{p}(n)
$$

By (4.65), we have

$$
\begin{aligned}
& \sum_{t=1}^{n} E\left(\left(E \psi^{2}\left(\frac{\eta_{1}}{\sigma}\right) \Delta_{n}(a, \sigma)\right)^{-1} \psi^{2}\left(\frac{\eta_{t}}{\sigma}\right) \varepsilon_{t-1}^{2} \mid H_{t-1}\right) \\
& =\left(E \psi^{2}\left(\frac{\eta_{1}}{\sigma}\right) \Delta_{n}(a, \sigma)\right)^{-1} \sum_{t=1}^{n} \varepsilon_{t-1}^{2} E\left(\psi^{2}\left(\frac{\eta_{t}}{\sigma}\right)\right) \\
& =\left(\Delta_{n}(a, \sigma)\right)^{-1} \sum_{t=1}^{n} \varepsilon_{t-1}^{2}=1+o_{p}(1) .
\end{aligned}
$$

For given $\delta>0$, there is a set whose probability approaches 1 as $n \rightarrow \infty$ on which $\max _{1 \leq t \leq n}\left|\frac{\varepsilon_{t}}{\sqrt{n}}\right| \leq \delta$. In this event, for any $c>0$,

$$
\begin{gathered}
\sum_{t=1}^{n} E\left\{\psi^{2}\left(\frac{\eta_{t}}{\sigma}\right) \frac{\varepsilon_{t-1}^{2}}{n} I\left(\left|\psi\left(\frac{\eta_{t}}{\sigma}\right) \frac{\varepsilon_{t-1}}{\sqrt{n}}\right|>c\right) \mid H_{t-1}\right\} \\
=\sum_{t=1}^{n} \int_{c}^{\infty} y^{2} d P\left\{\left|\psi\left(\frac{\eta_{t}}{\sigma}\right) \frac{\varepsilon_{t-1}}{\sqrt{n}}\right| \leq y \mid H_{t-1}\right\} \\
=\sum_{t=1}^{n} \frac{\varepsilon_{t-1}^{2}}{n} \int_{\frac{c}{\frac{\varepsilon_{t-1}}{\sqrt{n}}}}^{\infty} y^{2} d P\left\{\left|\psi\left(\frac{\eta_{t}}{\sigma}\right)\right| \leq y \mid H_{t-1}\right\} \\
\leq \sum_{t=1}^{n} \frac{\varepsilon_{t-1}^{2}}{n} \int_{\frac{c}{\delta}}^{\infty} y^{2} d P\left\{\left|\psi\left(\frac{\eta_{t}}{\sigma}\right)\right| \leq y \mid H_{t-1}\right\} \\
=o_{\delta} \sum_{t=1}^{n} \frac{\varepsilon_{t-1}^{2}}{n}=o_{\delta} O_{p}(1) \rightarrow 0, \quad n \rightarrow \infty .
\end{gathered}
$$

Here $o_{\delta} \rightarrow 0$ as $\delta \rightarrow 0$. This verifies the Lindeberg conditions, hence (4.63) follows from Lemma 4.5.

Note that $-\left[S_{n}(\theta)\right]_{\beta, \sigma}\left[\operatorname{Var}^{-\frac{1}{2}}\left(S_{n}(\theta)\right)\right]_{\beta, \sigma}$ are asymptotic independent of

$$
\sum_{t=1}^{n} \psi\left(\frac{\eta_{t}}{\sigma}\right) \varepsilon_{t-1}\left(E \psi^{2}\left(\frac{\eta_{1}}{\sigma}\right) \Delta_{n}(a, \sigma)\right)^{-\frac{1}{2}}
$$

Therefore, we obtain Theorem 3.1(1) from (4.61)-(4.63). 
(2) For $|a|=1$. By Lemma 4.5(2), Lemma 4.2(2), (2.9)-(2.11), (4.59), and $\theta \in \Theta_{2}$, we have

$$
\begin{aligned}
\left(\hat{\theta}_{n}-\theta\right) D_{n}(\theta)= & S_{n}(\theta)+\left(O_{p}(1), O_{p}\left(n^{\frac{1}{2}}\right)\right)+\left(O_{p}\left(n^{-\frac{1}{2}}\right), O_{p}\left(n^{\frac{1}{2}}\right)\right) \\
= & \left(\sum_{t=1}^{n} \psi\left(\frac{\eta_{t}}{\sigma}\right)\left(X_{t}-a X_{t-1}-\left(\zeta_{t}-a \zeta_{t-1}\right)\right)^{T},\right. \\
& \left.\sum_{t=1}^{n} \chi\left(\frac{\eta_{t}}{\sigma}\right)-A_{n}, \sum_{t=1}^{n} \psi\left(\frac{\eta_{t}}{\sigma}\right) \varepsilon_{t-1}\right)+\left(O_{p}(1), O_{p}\left(n^{\frac{1}{2}}\right)\right) \\
= & \left(\sum_{t=1}^{n} \psi\left(\frac{\eta_{t}}{\sigma}\right)\left(X_{t}-a X_{t-1}-\left(\zeta_{t}-a \zeta_{t-1}\right)\right)^{T},\right. \\
& \left.\sum_{t=1}^{n}\left(\chi\left(\frac{\eta_{t}}{\sigma}\right)-A\right), \sum_{t=1}^{n} \psi\left(\frac{\eta_{t}}{\sigma}\right) \varepsilon_{t-1}\right)+\left(O_{p}(1), O_{p}\left(n^{\frac{1}{2}}\right)\right) .
\end{aligned}
$$

Note that $\operatorname{Var}\left(S_{n}(\theta)\right)=\operatorname{diag}\left(O(n), O\left(n^{2}\right)\right)$, so we have

$$
\begin{aligned}
\left(\hat{\theta}_{n}-\theta\right) D_{n}(\theta) \operatorname{Var}^{-\frac{1}{2}}\left(S_{n}(\theta)\right)= & \left\{-\left[S_{n}(\theta)\right]_{\beta, \sigma}\left[\operatorname{Var}^{-\frac{1}{2}}\left(S_{n}(\theta)\right)\right]_{\beta, \sigma},\right. \\
& \left.\sum_{t=1}^{n} \psi\left(\frac{\eta_{t}}{\sigma}\right) \varepsilon_{t-1}\left(E \psi^{2}\left(\frac{\eta_{1}}{\sigma}\right) \Delta_{n}(a, \sigma)\right)^{-\frac{1}{2}}\right\} \\
& +o_{p}(1) .
\end{aligned}
$$

Similarly to the proof of (4.67) and (4.68), we have

$$
\sum_{t=1}^{n} E\left(\left(E \psi^{2}\left(\frac{\eta_{1}}{\sigma}\right) \Delta_{n}(a, \sigma)\right)^{-1} \psi^{2}\left(\frac{\eta_{t}}{\sigma}\right) \varepsilon_{t-1}^{2} \mid H_{t-1}\right)=1+o_{p}(1)
$$

and

$$
\sum_{t=1}^{n} E\left\{\psi^{2}\left(\frac{\eta_{t}}{\sigma}\right) \frac{\varepsilon_{t-1}^{2}}{n^{2}} I\left(\left|\psi\left(\frac{\eta_{t}}{\sigma}\right) \frac{\varepsilon_{t-1}}{n}\right|>c\right) \mid H_{t-1}\right\} \rightarrow 0, \quad n \rightarrow \infty .
$$

This verifies the Lindeberg conditions, hence (4.63) follows from Lemma 4.5. Similarly to the proof of Theorem 3.1(1), we easily prove Theorem 3.1(2).

(3) For $|a|>1$. By Lemma 4.3(3), Lemma 4.2(3), (2.9)-(2.11), (4.59), and $\theta \in \Theta_{3}$, we have

$$
\begin{aligned}
\left(\hat{\theta}_{n}-\theta\right) D_{n}(\theta)= & S_{n}(\theta)+\left(O_{p}(1), O_{p}\left(a^{n} n^{-\frac{1}{2}}\right)\right)+\left(O_{p}\left(n^{-\frac{1}{2}}\right), O_{p}\left(a^{n} n^{-1}\right)\right) \\
= & \left(\sum_{t=1}^{n} \psi\left(\frac{\eta_{t}}{\sigma}\right)\left(X_{t}-a X_{t-1}-\left(\zeta_{t}-a \zeta_{t-1}\right)\right)^{T}\right. \\
& \left.\sum_{t=1}^{n} \chi\left(\frac{\eta_{t}}{\sigma}\right)-A_{n}, \sum_{t=1}^{n} \psi\left(\frac{\eta_{t}}{\sigma}\right) \varepsilon_{t-1}\right)+\left(O_{p}(1), O_{p}\left(a^{n} n^{-\frac{1}{2}}\right)\right) \\
= & \left(\sum_{t=1}^{n} \psi\left(\frac{\eta_{t}}{\sigma}\right)\left(X_{t}-a X_{t-1}-\left(\zeta_{t}-a \zeta_{t-1}\right)\right)^{T}\right. \\
& \left.\sum_{t=1}^{n}\left(\chi\left(\frac{\eta_{t}}{\sigma}\right)-A\right), \sum_{t=1}^{n} \psi\left(\frac{\eta_{t}}{\sigma}\right) \varepsilon_{t-1}\right)+\left(O_{p}(1), O_{p}\left(a^{n} n^{-\frac{1}{2}}\right)\right) .
\end{aligned}
$$


Note that $\operatorname{Var}\left(S_{n}(\theta)\right)=\operatorname{diag}\left(O(n), O\left(a^{2 n}\right)\right)$, so we have

$$
\begin{aligned}
\left(\hat{\theta}_{n}-\theta\right) D_{n}(\theta) \operatorname{Var}^{-\frac{1}{2}}\left(S_{n}(\theta)\right)= & \left\{-\left[S_{n}(\theta)\right]_{\beta, \sigma}\left[\operatorname{Var}^{-\frac{1}{2}}\left(S_{n}(\theta)\right)\right]_{\beta, \sigma},\right. \\
& \left.\sum_{t=1}^{n} \psi\left(\frac{\eta_{t}}{\sigma}\right) \varepsilon_{t-1}\left(E \psi^{2}\left(\frac{\eta_{1}}{\sigma}\right) \Delta_{n}(a, \sigma)\right)^{-\frac{1}{2}}\right\} \\
& +o_{p}(1) .
\end{aligned}
$$

Similarly to the proof of Theorem 3.1(1), we easily prove Theorem 3.1(3). The proof of Theorem 3.1 is now complete.

\section{Proof of Theorem 3.2}

Case 1. For $|a|<1$. A first step towards (4.74) is to show that

$$
\begin{aligned}
& n^{-\frac{1}{2}} \sum_{t=1}^{n} \psi\left(\frac{\eta_{t}}{\sigma}\right)\left(X_{t}-a X_{t-1}-\left(\zeta_{t}-a \zeta_{t-1}\right)\right)^{T} \\
& \quad \rightarrow N\left(0,\left(n^{-1} X_{n}(a)+\left(1+a^{2}\right) \sigma_{\zeta}^{2} I_{d}\right) E\left(\psi^{2}\left(\frac{\eta_{1}}{\sigma}\right)\right)\right), \quad n \rightarrow \infty
\end{aligned}
$$

Let $u \in R^{d}$ with $|u|=1$. Then

$$
\begin{aligned}
s_{n}^{2} & =\operatorname{Var}\left\{n^{-\frac{1}{2}} \sum_{t=1}^{n} \psi\left(\frac{\eta_{t}}{\sigma}\right)\left(X_{t}-a X_{t-1}-\left(\zeta_{t}-a \zeta_{t-1}\right)\right)^{T} u\right\} \\
& =\left(n^{-1} u^{T} X_{n}(a) u+\left(1+a^{2}\right) \sigma_{\zeta}^{2}\right) E\left(\psi^{2}\left(\frac{\eta_{1}}{\sigma}\right)\right)=O(1) .
\end{aligned}
$$

Thus,

$$
\begin{aligned}
& s_{n}^{-(2+\delta)} \sum_{t=1}^{n} E\left|n^{-\frac{1}{2}} \psi\left(\frac{\eta_{t}}{\sigma}\right)\left(X_{t}-a X_{t-1}-\left(\zeta_{t}-a \zeta_{t-1}\right)\right)^{T} u\right|^{2+\delta} \\
& =s_{n}^{-(2+\delta)} n^{-\frac{2+\delta}{2}} E\left|\psi\left(\frac{\eta_{t}}{\sigma}\right)\right|^{2+\delta} \sum_{t=1}^{n}\left(\left|\left(X_{t}-a X_{t-1}\right)^{T} u\right|^{2+\delta}+E\left|\left(\zeta_{t}-a \zeta_{t-1}\right)^{T} u\right|^{2+\delta}\right) \\
& =O(1) n^{-\frac{\delta}{2}} E\left|\psi\left(\frac{\eta_{t}}{\sigma}\right)\right|^{2+\delta}\left(\max _{1 \leq t \leq n}\left|\left(X_{t}-a X_{t-1}\right)^{T} u\right|^{2+\delta}+O(1)\right) \\
& \rightarrow 0, \quad n \rightarrow \infty
\end{aligned}
$$

By Lemma 4.4 and the Cramer-Wold device, (4.75) follows from (4.77).

Next we need to show that

$$
n^{-\frac{1}{2}} \sum_{t=1}^{n}\left(\chi\left(\frac{\eta_{t}}{\sigma}\right)-A\right) \rightarrow N\left(0, \operatorname{Var}\left(\chi\left(\frac{\eta_{1}}{\sigma}\right)\right)\right), \quad n \rightarrow \infty
$$


In fact,

$$
\begin{aligned}
& \left(\operatorname{Var}\left(\chi\left(\frac{\eta_{1}}{\sigma}\right)\right)\right)^{-\frac{2+\delta}{2}} \sum_{t=1}^{n} E\left|n^{-\frac{1}{2}}\left(\chi\left(\frac{\eta_{t}}{\sigma}\right)-A\right)\right|^{2+\delta} \\
& \quad=\left(\operatorname{Var}\left(\chi\left(\frac{\eta_{1}}{\sigma}\right)\right)\right)^{-\frac{2+\delta}{2}} n^{-\frac{\delta}{2}} E\left|\chi\left(\frac{\eta_{t}}{\sigma}\right)-A\right|^{2+\delta} \\
& \quad \rightarrow 0, \quad n \rightarrow \infty .
\end{aligned}
$$

By Lemma 4.4, (4.78) follows from (4.79).

Finally, by (4.63), we easily prove that

$$
n^{-\frac{1}{2}} \sum_{t=1}^{n} \psi\left(\frac{\eta_{t}}{\sigma}\right) \varepsilon_{t-1} \rightarrow N\left(0, \frac{\sigma^{2}}{1-a^{2}} E\left(\psi^{2}\left(\frac{\eta_{1}}{\sigma}\right)\right)\right), \quad n \rightarrow \infty
$$

Case 2. For $|a| \geq 1$. Similarly to the proof of Case 1 , we easily prove this case.

This completes the proof of Theorem 3.2.

\section{Numerical example}

In the section, we will simulate a simple linear regression model (1.1) with (1.2), where $X_{t}=10 \cos \left(\frac{2 \pi t}{50}\right), \beta=3, n=50, \zeta_{t}$ and $\eta_{t} \sim N(0,1)$.

We take $\rho(u)=u(2 \Phi(u)-1)+2 \Phi(u)-\frac{2}{\sqrt{2 \pi}}, \psi(u)=2 \Phi(u)-1, \chi(u)=\frac{2}{\sqrt{2 \pi}}-2 \Phi^{\prime}(u)$, $A_{n}=\frac{(\sqrt{2}-1) n}{\sqrt{\pi}}$, where $\Phi(\cdot)$ and $\Phi^{\prime}(\cdot)$ are the distribution and density function of standard normal $N(0,1)$, respectively $\mathrm{Hu}[1]$. In the following, we calculate by using our method and the quasi-Newton line search method.

Case 1. For $a=0.5$, we have $\hat{\beta}_{n}=3.1125, \hat{a}_{n}=0.4074$, and $\hat{\sigma}_{n}=0.9940 . \hat{\beta}_{n}$ and $\hat{\sigma}_{n}$ approximately equal $\beta$ and $\sigma$, respectively.

Case 2. For $a=-1$, we have $\hat{\beta}_{n}=3.0766, \hat{a}_{n}=-1.0000$ and $\hat{\sigma}_{n}=0.5311$. For $a=1$, we have $\hat{\beta}_{n}=3.0572, \hat{a}_{n}=1.0000$, and $\hat{\sigma}_{n}=0.5298 . \hat{\beta}_{n}$ and $\hat{a}_{n}$ approximately equal $\beta$ and $a$, respectively.

Case 3. For $a=1.1$, we have $\hat{\beta}_{n}=3.0710, \hat{a}_{n}=1.1080$, and $\hat{\sigma}_{n}=0.5307 . \hat{\beta}_{n}$ and $\hat{a}_{n}$ approximately equal $\beta$ and $a$, respectively.

The above results show that our estimation method is valid in some cases.

\section{Competing interests}

The authors declare that they have no competing interests.

\section{Authors' contributions}

All authors contributed equally to the writing of this paper. All authors read and approved the final manuscript.

\section{Author details}

'School of Mathematics and Statistics, Hubei Normal University, Huangshi, 435002, China. ${ }^{2}$ Faculty of Information Engineering, China University Geosciences, Wuhan, 430074, China.

\section{Acknowledgements}

The first author's work was supported by the Natural Science Foundation of China (No. 11471105). The second author's work was Supported by Natural Science Foundation of China (No. 41374017). 


\section{References}

1. $\mathrm{Hu}, \mathrm{HH}$ : Asymptotic normality of Huber-Dutter estimators in a linear model with AR(1) processes. J. Stat. Plan. Inference 143(3), 548-562 (2013)

2. Maller, RA: Asymptotics of regressions with stationary and nonstationary residuals. In: Stochastic Processes and Their Appliations, vol. 105, pp. 33-67 (2003)

3. Pere, P: Adjusted estimates and Wald statistics for the AR(1) model with constant. J. Econom. 98, 335-363 (2000)

4. Fuller, WA: Introduction to Statistical Time Series, 2nd edn. Wiley, New York (1996)

5. Miao, Y, Liu, W: Moderate deviations for LS estimator in simple linear EV regression model. J. Stat. Plan. Inference 139(9), 3122-3131 (2009)

6. Miao, Y, Wang, K, Zhao, F: Some limit behaviors for the LS estimator in simple linear EV regression models. Stat. Probab. Lett. 81, 92-102 (2011)

7. Miao, Y, Yang, G, Shen, L: The central limit theorem for LS estimator in simple linear EV regression models. Commun Stat., Theory Methods 36, 2263-2272 (2007)

8. Liu, JX, Chen, XR: Consistency of LS estimator in simple linear EV regression models. Acta Math. Sci. 25, 50-58 (2005)

9. Cui, HJ: Asymptotic normality of M-estimates in the EV model. J. Syst. Sci. Math. Sci. 10(3), 225-236 (1997)

10. Cui, HJ, Chen, SX: Empirical likelihood confidence region for parameter in the errors-in-variables models. J. Multivar. Anal. 84, 101-115 (2003)

11. Cheng, CL, Van Ness, JW: Statistical Regression with Measurement Error. Arnold, London (1999)

12. Hamilton, JD: Time Series Analysis. Princeton University Press, Princeton (1994)

13. Brockwell, PJ, Davis, RA: Time Series: Theory and Methods. Springer, New York (1987)

14. Baran, S: A consistent estimator for linear models with dependent observations. Commun. Stat., Theory Methods 33(10), 2469-2486 (2004)

15. Fan, GL, Liang, HY, Wang, JF, Xu, HX: Asymptotic properties for LS estimators in EV regression model with dependent errors. AStA Adv. Stat. Anal. 94, 89-103 (2010)

16. Miao, Y, Zhao, F, Wang, K: Central limit theorems for LS estimators in the EV regression model with dependent measurements. J. Korean Stat. Soc. 40, 303-312 (2011)

17. Fuller, WA: Measurement Error Models. Wiley, New York (1987)

18. Brown, M: Robust line estimation with errors in both variables. J. Am. Stat. Assoc. 77, 71-79 (1982)

19. Ketellapper, $\mathrm{RH}$, Ronner, AE: Are robust estimation methods useful in the structural errors-in-variables model. Metrika 31, 33-41 (1984)

20. Zamar, RH: Robust estimation in the errors-in-variables model. Biometrika 76(1), 149-160 (1989)

21. Cheng, CL, Van Ness, JW: Generalized M-estimators for errors-in-variables regression. Ann. Stat. 20(1), 385-397 (1992)

22. $\mathrm{He}, \mathrm{X}$, Liang, $\mathrm{H}$ : Quantile regression estimates for a class of linear and partially linear errors-in-variables models. Stat. Sin. 10, 129-140 (2000)

23. Fekri, M, Ruiz-Gazen, A: Robust weighted orthogonal regression in the errors-in-variables model. J. Multivar. Anal. 88 , 89-108 (2004)

24. Wu, WB: M-Estimation of linear models with dependent errors. Ann. Stat. 35(2), 495-521 (2007)

25. Silvapullé, MJ: Asymptotic behavior of robust estimators of regression and scale parameters with fixed carriers. Ann. Stat. 13(4), 1490-1497 (1985)

26. Hampel, FR, Ronchetti, EM, Rousseeuw, PJ, et al.: Robust Statistics. Wiley, New York (1986)

27. Huber, PJ, Ronchetti, EM: Robust Statistics, 2nd edn. Wiley, New York (2009)

28. Li, L: On Koul's minimum distance estimators in the regression models with long memory moving averages. In: Stochastic Processes and Their Applications, vol. 105, pp. 257-269 (2003)

29. Babu, GJ: Strong representations for LAD estimators in linear models. Probab. Theory Relat. Fields 83, 547-558 (1989)

30. Salibian-Barrera, M, Zamar, RH: Bootstrapping robust estimates of regression. Ann. Stat. 30(2), 556-582 (2002)

31. Wu, R, Wang, Q: Shrinkage estimation for linear regression with ARMA errors. J. Stat. Plan. Inference 142, 2136-2148 (2012)

32. Zhou, Z, Wu, W: On linear models with long memory and heavy-tailed errors. J. Multivar. Anal. 102, 349-362 (2011)

33. Tong, XW, Cui, HJ, Yu, P: Consistency and normality of Huber-Dutter estimators for partial linear model. Sci. China Ser. A 51(10), 1831-1842 (2008)

34. Arcones, MA: The Bahadur-Kiefer representation of $L_{p}$ regression estimators. Econom. Theory 12, $257-283$ (1996)

35. Zeckhauser, R, Thompson, M: Linear regression with non-normal error terms. Rev. Econ. Stat. 52, 280-286 (1970)

36. Ronner, AE: P-norm estimators in a linear regression model. Ph.D. thesis, Drukkerijenbv-Groningen, The Netherlands (1977)

37. Ronner, AE: Asymptotic normality of p-norm estimators in multiple regression. Z. Wahrscheinlichkeitstheor. Verw. Geb. 66, 613-620 (1984)

38. Prakasa Rao, BLS: Asymptotic Theory of Statistical Inference. Wiley, New York (1987)

39. Hall, P, Heyde, CC: Martingale Limit Theory and Its Application. Academic Press, New York (1980)

10.1186/1029-242X-2014-474

Cite this article as: Hu and Pan: Asymptotic normality of Huber-Dutter estimators in a linear EV model with AR(1) processes. Journal of Inequalities and Applications 2014, 2014:474 